\title{
HILL'S EQUATION WITH RANDOM FORCING TERMS
}

\author{
Fred C. Adams ${ }^{1,2}$ and Anthony M. Bloch ${ }^{1,3}$ \\ ${ }^{1}$ Michigan Center for Theoretical Physics \\ Physics Department, University of Michigan, Ann Arbor, MI 48109 \\ ${ }^{2}$ Astronomy Department, University of Michigan, Ann Arbor, MI 48109, fca@umich.edu, phone: \\ (734)647-4320, fax: (734)763-0937 \\ ${ }^{3}$ Department of Mathematics, University of Michigan, Ann Arbor, MI 48109, abloch@umich.edu, \\ phone (734)647-4980, fax:(734)763-0937
}

\begin{abstract}
Motivated by a class of orbit problems in astrophysics, this paper considers solutions to Hill's equation with forcing strength parameters that vary from cycle to cycle. The results are generalized to include period variations from cycle to cycle. The development of the solutions to the differential equation is governed by a discrete map. For the general case of Hill's equation in the unstable limit, we consider separately the case of purely positive matrix elements and those with mixed signs; we then find exact expressions, bounds, and estimates for the growth rates. We also find exact expressions, estimates, and bounds for the infinite products of several $2 \times 2$ matrices with random variables in the matrix elements. In the limit of sharply spiked forcing terms (the delta function limit), we find analytic solutions for each cycle and for the discrete map that matches solutions from cycle to cycle; for this case we find the growth rates and the condition for instability in the limit of large forcing strength, as well as the widths of the stable/unstable zones.
\end{abstract}

Keywords: Hill's equation, random matrices, orbit problems.

AMS subject classifcation: 34C11, 15A52

\section{INTRODUCTION}

This paper presents new results concerning Hill's equation of the form

$$
\frac{d^{2} y}{d t^{2}}+\left[\lambda_{k}+q_{k} \hat{Q}(t)\right] y=0
$$

where the function $\hat{Q}(t)$ is periodic, so that $\hat{Q}(t+\pi)=\hat{Q}(t)$, and normalized, so that $\int_{0}^{\pi} \hat{Q} d t=$ 1. The parameter $q_{k}$ is denoted here as the forcing strength, which we consider to be a random variable that takes on a new value every cycle (the index $k$ determines the cycle). The parameter $\lambda_{k}$, which determines the oscillation frequency in the absence of forcing, also varies from cycle to 
cycle. In principal, the duration of the cycle could also vary; our first result (see Theorem 1) shows that this generalized case can be reduced to the problem of equation (1).

Hill's equations $[\mathrm{HI}]$ arise in a wide variety of contexts $[\mathrm{MW}]$ and hence the consideration of random variations in the parameters $\left(q_{k}, \lambda_{k}\right)$ is a natural generalization of previous work. This particular form of Hill's equation was motivated by a class of orbit problems in astrophysics [AB]. In many astrophysical systems, orbits take place in extended mass distributions with triaxial forms. Examples include dark matter halos that envelop galaxies and galaxy clusters, stellar bulges found at the centers of spiral galaxies, elliptical galaxies, and young embedded star clusters. These systems thus occur over an enormous range of scales, spanning factors of millions in size and factors of trillions in mass. Nonetheless, the basic form of the potential is similar [NF, BE, AB] for all of these systems, and the corresponding orbit problem represents a sizable fraction of the orbital motion that takes place in our universe. In this context, when a test particle (e.g., a star or a dark matter particle) orbits within the triaxial potential, motion that is initially confined to a particular orbital plane can be unstable to motion in the perpendicular direction [AB]. The equation that describes the development of this instability takes the form of equation (1). Further, the motion in the original orbital plane often displays chaotic behavior, which becomes more extreme as the axis ratios of the potential increase $[\mathrm{BT}]$. In this application, the motion in the original orbit plane - in particular, the distance to the center of the coordinate system - determines the magnitude of the forcing strength $q_{k}$ that appears in Hill's equation. The crossing time, which varies from orbit to orbit, determines the value of the oscillation parameter $\lambda_{k}$. As a result, the chaotic behavior in the original orbital plane leads to random forcing effects in the differential equation that determines instability of motion out of the plane (see Appendix A for further discussion).

Given that Hill's equations arise in a wide variety of physical problems [MW], we expect that applications with random forcing terms will be common. Although the literature on stochastic differential equations is vast (e.g., see the review of $[\mathrm{BL}]$ ), specific results regarding Hill's equations with random forcing terms are relatively rare.

In this application, Hill's equation is periodic or nearly periodic (we generalize to the case of varying periods for the basic cycles), and the forcing strength $q_{k}$ varies from cycle to cycle. Since the forcing strength is fixed over a given cycle, one can solve the Hill's equation for each cycle using previously developed methods $[\mathrm{MW}]$, and then match the solutions from cycle to cycle using a discrete map. As shown below, the long-time solution can be developed by repeated multiplication of $2 \times 2$ matrices that contain a random component in their matrix elements.

The subject of random matrices, including the long term behavior of their products, is also the subject of a great deal of previous work [BL, DE, BD, FK, FU, LR, ME, VI]. In this application, however, Hill's equation determines the form of the random matrices and the repeated multiplication of this type of matrix represents a new and specific application. Given that instances where analytic results can be obtained are relatively rare, this set of solutions adds new examples to the list of known cases. 
This paper is organized as follows. In $\S 2$, we present the basic formulation of the problem, define relevant quantities, and show that aperiodic generalizations of the problem can be reduced to random Hill's equations. The following section ( 33 ) presents the main results of the paper: We find specific results regarding the growth rates of instability for random Hill's equations in the limit of large forcing strengths (i.e., in the limit where the equations are robustly unstable). These results are presented for purely positive and for mixed signs in the $2 \times 2$ matrix map. We also find limiting forms and constraints on the growth rates. Finally, we find bounds and estimates for the errors incurred by working in the limit of large forcing strengths. This work is related to the general existence results of [FU] but provides much more detailed information in our setting. In the next section $(\S 4)$ we consider the limit where the forcing terms are Dirac delta functions; this case allows for analytic solutions to the original differential equation. We note that the growth rates calculated here $(\S 3)$ depend on the distribution of the ratios of the principal solutions to equation (1), rather than (directly) on the distributions of the parameters $\left(\lambda_{k}, q_{k}\right)$. Using the analytic solutions for the delta function limit $(\S 4)$, we thus gain insight into the transformation between the distributions of the input parameters $\left(\lambda_{k}, q_{k}\right)$ and the parameters that specify the growth rates. Finally, we conclude, in $\S 5$, with a summary and discussion of our results.

\section{FORMULATION}

Definition: A Random Hill's equation is defined here to be of the form given by equation (1) where the forcing strength $q_{k}$ and oscillation parameter $\lambda_{k}$ vary from cycle to cycle. Specifically, the parameters $q_{k}$ and $\lambda_{k}$ are stochastic variables that take on new values every cycle $0 \leq[t] \leq \pi$, and the values are sampled from known probability distributions $P_{q}(q)$ and $P_{\lambda}(\lambda)$.

\subsection{Hill's Equation with Fixed Parameters}

Over a single given cycle, a random Hill's equation is equivalent to an ordinary Hill's equation and can be solved using known methods [MW].

Definition: The growth factor $f_{c}$ per cycle (the Floquet multiplier) is given by the solution to the characteristic equation and can be written as

$$
f_{c}=\frac{\Delta+\left(\Delta^{2}-4\right)^{1 / 2}}{2}
$$

where the discriminant $\Delta=\Delta(q, \lambda)$ is defined by

$$
\Delta \equiv y_{1}(\pi)+\frac{d y_{2}}{d t}(\pi)
$$

and where $y_{1}$ and $y_{2}$ are the principal solutions [MW].

It follows from Floquet's Theorem that $|\Delta|>2$ is a sufficient condition for instability [MW, AS]. In addition, periodic solutions exist when $|\Delta|=2$. 


\subsection{Random Variations in Forcing Strength}

We now generalize to the case where the forcing strength $q_{k}$ and oscillation parameter $\lambda_{k}$ vary from cycle to cycle. In other words, we consider each period from $t=0$ to $t=\pi$ as a cycle, and consider the effects of successive cycles with varying values of $\left(q_{k}, \lambda_{k}\right)$.

During any given cycle, the solution can be written as a linear combination of the two principal solutions $y_{1}$ and $y_{2}$. Consider two successive cycles. The first cycle has parameters $\left(q_{a}, \lambda_{a}\right)$ and solution

$$
f_{a}(t)=\alpha_{a} y_{1 a}(t)+\beta_{a} y_{2 a}(t),
$$

where the solutions $y_{1 a}(t)$ and $y_{2 a}(t)$ correspond to those for an ordinary Hill's equation when evaluated using the values $\left(q_{a}, \lambda_{a}\right)$. Similarly, for the second cycle with parameters $\left(q_{b}, \lambda_{b}\right)$ the solution has the form

$$
f_{b}(t)=\alpha_{b} y_{1 b}(t)+\beta_{b} y_{2 b}(t)
$$

Next we note that the new coefficients $\alpha_{b}$ and $\beta_{b}$ are related to those of the previous cycle through the relations

$$
\alpha_{b}=\alpha_{a} y_{1 a}(\pi)+\beta_{a} y_{2 a}(\pi) \quad \text { and } \quad \beta_{b}=\alpha_{a} \frac{d y_{1 a}}{d t}(\pi)+\beta_{a} \frac{d y_{2 a}}{d t}(\pi) .
$$

The new coefficients can thus be considered as a two dimensional vector, and the transformation between the coefficients in one cycle and the next cycle is a $2 \times 2$ matrix. Here we consider the case in which the equation is symmetric with respect to the midpoint $t=\pi / 2$. This case arises in the original orbit problem that motivated this study - the forcing function is determined by the orbit as it passes near the center of the potential and this passage is symmetric (or very nearly so). It also makes sense to consider the symmetric case, which is easier, first. Since the Wronskian of the original differential equation is unity, the number of independent matrix coefficients is reduced further, from four to two. We thus have the following result:

Proposition 1: The transformation between the coefficients $\alpha_{a}, \beta_{a}$ of one cycle and the coefficients $\alpha_{b}, \beta_{b}$ of the next may be written in the form

$$
\left[\begin{array}{c}
\alpha_{b} \\
\beta_{b}
\end{array}\right]=\left[\begin{array}{cc}
h & \left(h^{2}-1\right) / g \\
g & h
\end{array}\right]\left[\begin{array}{c}
\alpha_{a} \\
\beta_{a}
\end{array}\right] \equiv \mathbf{M}\left(q_{a}\right)\left[\begin{array}{c}
\alpha_{a} \\
\beta_{a}
\end{array}\right]
$$

where the matrix $\mathbf{M}$ (defined in the second equality) depends on the values $\left(q_{a}, \lambda_{a}\right)$ and $h=y_{1}(\pi)$ and $g=\dot{y}_{1}(\pi)$ for a given cycle.

Proof: This result can be verified by standard matrix multiplication, which yields equation (6) above.

After $N$ cycles with varying values of $\left(q_{k}, \lambda_{k}\right)$, the solution retains the general form given above, where the coefficients are determined by the product of matrices according to

$$
\left[\begin{array}{c}
\alpha_{N} \\
\beta_{N}
\end{array}\right]=\mathbf{M}^{(N)}\left[\begin{array}{c}
\alpha_{0} \\
\beta_{0}
\end{array}\right] \quad \text { where } \quad \mathbf{M}^{(N)} \equiv \prod_{k=1}^{N} \mathbf{M}_{k}\left(q_{k}, \lambda_{k}\right) .
$$


This formulation thus transforms the original differential equation (with a random element) into a discrete map. The properties of the product matrix $\mathbf{M}^{(N)}$ determine whether the solution is unstable and the corresponding growth rate.

Definition: The asymptotic growth rate $\gamma_{\infty}$ is that experienced by the system when each cycle amplifies the growing solution by the growth factor appropriate for the given value of the forcing strength for that cycle, i.e.,

$$
\gamma_{\infty} \equiv \lim _{N \rightarrow \infty} \frac{1}{\pi N} \log \left[\prod_{k=1}^{N} \frac{1}{2}\left\{\Delta_{k}+\sqrt{\Delta_{k}^{2}-4}\right\}\right],
$$

where $\Delta_{k}=\Delta\left(q_{k}, \lambda_{k}\right)$ is defined by equation (3), and where this expression is evaluated in the limit $N \rightarrow \infty$. In this definition, it is understood that if $\left|\Delta_{k}\right|<2$ for a particular cycle, then the growth factor is unity for that cycle, resulting in no net contribution to the product (for that cycle).

Notice that the factor of $\pi$ appears in this definition of the growth rate because the original Hill's equation is taken to be $\pi$-periodic [MW, AS]. As we show below, the growth rates of the differential equation are determined by the growth rates resulting from matrix multiplication. In many cases, however, the growth rates for matrix multiplication are given without the factor of $\pi$ $[\mathrm{BL}, \mathrm{FK}]$, so there is a mismatch of convention (by a factor of $\pi$ ) between growth rates of Hill's equations and growth rates of matrix multiplication.

Notice that this expression for the asymptotic growth rate takes the form

$$
\gamma_{\infty}=\lim _{N \rightarrow \infty} \frac{1}{N} \sum_{k=1}^{N} \gamma\left(q_{k}, \lambda_{k}\right) \rightarrow\langle\gamma\rangle
$$

where $\gamma\left(q_{k}, \lambda_{k}\right)$ is the growth rate for a given cycle. The asymptotic growth rate is thus given by the expectation value of the growth rate per cycle for a given probability distribution for the parameters $q_{k}$ and $\lambda_{k}$.

We note that a given system does not necessarily experience growth at the rate $\gamma_{\infty}$ because the solutions must remain continuous across the boundaries between subsequent cycles. This requirement implies that the solutions during every cycle will contain an admixture of both the growing solution and the decaying solution for that cycle, thereby leading to the possibility of slower growth. In some cases, however, the growth rate is larger than $\gamma_{\infty}$, i.e., the stochastic component of the problem aids and abets the instability. One could also call $\gamma_{\infty}$ the direct growth rate.

\subsection{Generalization to Aperiodic Variations}

Theorem 1: Consider a generalization of Hill's equation so that the cycles are no longer exactly $\pi$-periodic. Instead, each cycle has period $\mu_{k} \pi$, where $\mu_{k}$ is a random variable that averages to unity. Then variations in period are equivalent to variations in $(q, \lambda)$, i.e., the problem with three 
stochastic variables $\left(q_{k}, \lambda_{k}, \mu_{k}\right)$ reduces to a $\pi$-periodic problem with only two stochastic variables $\left(q_{k}, \lambda_{k}\right)$.

Proof: With this generalization, the equation of motion takes the form

$$
\frac{d^{2} y}{d t^{2}}+\left[\lambda_{k}+q_{k} \hat{Q}\left(\mu_{k} t\right)\right] y=0
$$

where we have normalized the forcing frequency to have unit amplitude $\left(\hat{Q}=Q / q_{k}\right)$. Since $\hat{Q}$ (and $Q)$ are $\pi$-periodic, the $j t h$ cycle is defined over the time interval $0 \leq \mu_{k} t \leq \pi$, or $0 \leq t \leq \pi / \mu_{k}$. We can re-scale both the time variable and the "constants" according to

$$
t \rightarrow \mu_{k} t, \quad \lambda_{k} \rightarrow \lambda_{k} / \mu_{k}^{2}=\widetilde{\lambda}_{j}, \quad \text { and } \quad q_{k} \rightarrow q_{k} / \mu_{k}^{2}=\widetilde{q}_{j},
$$

so the equation of motion reduces to the familiar form

$$
\frac{d^{2} y}{d t^{2}}+\left[\widetilde{\lambda}_{j}+\widetilde{q}_{j} \hat{Q}(t)\right] y=0
$$

Thus, the effects of varying period can be incorporated into variations in the forcing strength $q_{k}$ and oscillation parameter $\lambda_{k}$.

\section{HILL'S EQUATION IN THE UNSTABLE LIMIT}

In this section we consider Hill's equation in general form (for the delta function limit see $\S 4$ ), but restrict our analysis to the case of symmetric potentials so that $y_{1}(\pi)=h=\dot{y}_{2}(\pi)$. We also consider the highly unstable limit, where we define this limit to correspond to large $h \gg 1$. Since the $2 \times 2$ matrix of the discrete map must have its determinant equal to unity, the matrix of the map has the form given by equation (7), where the values of $h$ and $g$ depend on the form of the forcing potential.

The discrete map can be rewritten in the general form

$$
\mathbf{M}=h\left[\begin{array}{cc}
1 & x \\
1 / x & 1
\end{array}\right]+\left[\begin{array}{cc}
0 & -1 / g \\
0 & 0
\end{array}\right]
$$

In the highly unstable limit $h \rightarrow \infty$, the matrix simplifies to the approximate form

$$
\mathbf{M} \approx h\left[\begin{array}{cc}
1 & x \\
1 / x & 1
\end{array}\right] \equiv h \mathbf{C},
$$

where we have defined $x \equiv h / g$, and where the second equality defines the matrix $\mathbf{C}$.

In this problem we are concerned with both the long-time limit $N \rightarrow \infty$, and the "unstable" limit $h \rightarrow \infty$. In the first instance considered here we take the unstable limit first, but below we analyze precisely the difference between taking the long time limit first and then the unstable limit. 


\subsection{Fixed Matrix of the Discrete Map}

The simplest case occurs when the stochastic component can be separated from the matrix, i.e., when the matrix $\mathbf{C}$ does not vary from cycle to cycle. This case arises when the Hill's equation does not contain a random component; it also arises when the random component can be factored out so that $x$ does not vary from cycle to cycle, although the leading factors $h_{k}$ can vary. In either case, the matrix $\mathbf{C}$ is fixed. Repeated multiplications of the matrix $\mathbf{C}$ are then given by

$$
\mathbf{C}^{N}=2^{N-1} \mathbf{C} .
$$

With this result, after $N$ cycles the Floquet multiplier (eigenvalue) of the product matrix and the corresponding growth rate take the form

$$
\Lambda=\prod_{k=1}^{N}\left(2 h_{k}\right) \quad \text { and } \quad \gamma=\lim _{N \rightarrow \infty} \frac{1}{\pi N} \sum_{k=1}^{N} \log \left(2 h_{k}\right) .
$$

Note that this result applies to the particular case of Hill's equation in the delta function limit $(\S 4)$, where the forcing strength $q_{k}$ varies from cycle to cycle but the frequency parameter $\lambda_{k}$ is constant. The growth rate in equation (17) is equal to the asymptotic growth rate $\gamma_{\infty}$ (eq. [9]) for this case.

\subsection{General Results in the Unstable Limit}

We now generalize to the case where the parameters of the differential equation vary from cycle to cycle. For a given cycle, the discrete map is specified by a matrix of the form specified by equation (15), where $x=x_{k}=h_{k} / g_{k}$, with varying values from cycle to cycle. The values of $x_{k}$ depend on the parameters $\left(q_{k}, \lambda_{k}\right)$ through the original differential equation. After $N$ cycles, the product matrix $\mathbf{M}^{(N)}$ takes the form

$$
\mathbf{M}^{(N)}=\prod_{k=1}^{N} h_{k} \prod_{k=1}^{N} \mathbf{C}_{k}
$$

where we have separated out the two parts of the problem. One can show (by induction) that the product of $N$ matrices $\mathbf{C}_{k}$ have the form

$$
\mathbf{C}^{(N)}=\prod_{k=1}^{N} \mathbf{C}_{k}=\left[\begin{array}{cc}
\Sigma_{T(N)} & x_{1} \Sigma_{T(N)} \\
\Sigma_{B(N)} / x_{1} & \Sigma_{B(N)}
\end{array}\right],
$$

where $x_{1}$ is the value of the variable for the first cycle and where the sums $\Sigma_{T(N)}$ and $\Sigma_{B(N)}$ are given by

$$
\Sigma_{T(N)}=\sum_{j=1}^{2^{N-1}} r_{j} \quad \text { and } \quad \Sigma_{B(N)}=\sum_{j=1}^{2^{N-1}} \frac{1}{r_{j}}
$$


where the variables $r_{j}$ are ratios of the form

$$
r_{j}=\frac{x_{a_{1}} x_{a_{2}} \ldots x_{a_{n}}}{x_{b_{1}} x_{b_{2}} \ldots x_{b_{n}}}
$$

The ratios $r_{j}$ arise from repeated multiplication of the matrices $\mathbf{C}_{k}$, and hence the indices lie in the range $1 \leq a_{i}, b_{i} \leq N$. The $r_{j}$ always have the same number of factors in the numerator and the denominator, but the number of factors $(n)$ varies from 0 (where $r_{j}=1$ ) up to $N / 2$. This upper limit arises because each composite ratio $r_{j}$ has $2 n$ values of $x_{j}$, which must all be different, and because the total number of possible values is $N$.

Next we define a composite variable

$$
\widetilde{S} \equiv \frac{1}{2^{N}}\left[\Sigma_{T(N)}+\Sigma_{B(N)}\right]=\frac{1}{2^{N}} \sum_{j=1}^{2^{N-1}}\left(r_{j}+\frac{1}{r_{j}}\right) .
$$

With this definition, the (growing) eigenvalue $\Lambda$ of the product matrix $\mathbf{M}^{(N)}$ takes the form

$$
\Lambda=\widetilde{S} \prod_{k=1}^{N}\left(2 h_{k}\right)
$$

and the corresponding growth rate of the instability has the form

$$
\gamma=\lim _{N \rightarrow \infty}\left[\frac{1}{\pi N} \sum_{k=1}^{N} \log \left(2 h_{k}\right)+\frac{1}{N \pi} \log \widetilde{S}\right] .
$$

The first term is the asymptotic growth rate $\gamma_{\infty}$ defined by equation (9) and is thus an average of the growth rates for the individual cycles. All of the additional information regarding the stochastic nature of the differential equation is encapsulated in the second term through the variable $\widetilde{S}$. For example, if the composite variable $\widetilde{S}$ is finite in the limit $N \rightarrow \infty$, then the second term would vanish. As shown below, however, the stochastic component can provide a significant contribution to the growth rate, and can provide either a stabilizing or destabilizing influence. In the limit $N \rightarrow \infty$, we can thus write the growth rate in the from

$$
\gamma=\gamma_{\infty}+\Delta \gamma
$$

where we have defined the correction term $\Delta \gamma$,

$$
\Delta \gamma \equiv \lim _{N \rightarrow \infty} \frac{1}{N \pi} \log \widetilde{S}
$$

Since the asymptotic growth rate $\gamma_{\infty}$ is straightforward to evaluate, the remainder of this section focuses on evaluating $\Delta \gamma$, as well as finding corresponding estimates and constraints. This correction term $\Delta \gamma$ is determined by the discrete map $\mathbf{C}$, whose matrix elements are given by the ratios $x=h / g$, where $h$ and $g$ are determined by the solutions to Hill's equation over one cycle. 
One should keep in mind that the parameters in the original differential equation are $\left(\lambda_{k}, q_{k}\right)$. The distribution of these parameters determines the distributions of the principal solutions (the distributions of $h_{k}$ and $g_{k}$ ), whereas the distribution of the ratios $x_{k}$ of these latter quantities determines the correction $\Delta \gamma$ to the growth rate. The problem thus separates into two parts: [1] The transformation between the distributions of the parameters $\left(\lambda_{k}, q_{k}\right)$ and the resulting distribution of the ratios $x_{k}$ that define the discrete map, and [2] The growth rate of the discrete map for a given distribution of $x_{k}$. The following analysis focuses on the latter issue (whereas $\S 4$ provides an example of the former issue).

\subsection{Growth Rates for Positive Matrix Elements}

This subsection addresses the cases where the ratios $x_{k}$ that define the discrete map $\mathbf{C}$ all have the same sign. For this case, the analysis is simplified, and a number of useful results can be obtained.

Theorem 2: Consider the general form of Hill's equation in the unstable limit so that $h=y_{1}(\pi)=$ $\dot{y}_{2}(\pi) \gg 1$. For the case of positive matrix elements, $r_{j}>0$, the growth rate is given by equation (25) where the correction term $\Delta \gamma$ is given by

$$
\Delta \gamma=\lim _{N \rightarrow \infty} \frac{1}{\pi N} \sum_{j=1}^{N} \log \left(1+x_{j 1} / x_{j 2}\right)-\frac{\log 2}{\pi},
$$

where $x_{j 1}$ and $x_{j 2}$ represent two different (independent) samples of the $x_{j}$ variable. $^{1}$

Proof: Using the same induction argument that led to equation (19), one finds that from one cycle to the next the sums $\Sigma_{T(N)}$ and $\Sigma_{B(N)}$ vary according to

$$
\Sigma_{T(N+1)}=\Sigma_{T(N)}+\frac{x}{x_{0}} \Sigma_{B(N)}
$$

and

$$
\Sigma_{B(N+1)}=\Sigma_{B(N)}+\frac{x_{0}}{x} \Sigma_{T(N)}
$$

In this notation, the variable $x$ (no subscript) represents the value of the $x$ variable at the current cycle, whereas $x_{0}$ represents the value at the initial cycle. The growing eigenvalue of the product matrix of equation (19) is given by $\Lambda=\Sigma_{T(N)}+\Sigma_{B(N)}$. As a result, the eigenvalue (growth factor) varies from cycle to cycle according to

$$
\Lambda^{(N+1)}=\Lambda^{(N)}+\frac{x}{x_{0}} \Sigma_{B(N)}+\frac{x_{0}}{x} \Sigma_{T(N)}=\Lambda^{(N)}\left[1+\frac{\left(x / x_{0}\right) \Sigma_{B(N)}+\left(x_{0} / x\right) \Sigma_{T(N)}}{\Sigma_{B(N)}+\Sigma_{T(N)}}\right] .
$$

\footnotetext{
${ }^{1}$ Specifically, the index $j$ labels the cycle number, and the indices $j 1$ and $j 2$ label two successive samples of the $x$ variable; since the stochastic parameters of the differential equations are assumed to be independent from cycle to cycle, however, the variables $x_{j 1}$ and $x_{j 2}$ can be any independent samples.
} 
The overall growth factor is then determined by the product

$$
\Lambda^{(N)}=\prod_{j=1}^{N}\left[1+\frac{\left(x / x_{0}\right) \Sigma_{B(N)}+\left(x_{0} / x\right) \Sigma_{T(N)}}{\Sigma_{B(N)}+\Sigma_{T(N)}}\right] .
$$

The growth rate of matrix multiplication is determined by setting the above product equal to $\exp [N \pi \gamma]$. The growth rate $\Delta \gamma$ also includes the factor of 2 per cycle that is included in the definition of the asymptotic growth rate $\gamma_{\infty}$. We thus find that

$$
\Delta \gamma \approx \frac{1}{N \pi} \sum_{j=1}^{N} \log \left[1+\frac{\left(x_{j 1} / x_{j 2}\right) \Sigma_{B(N)}+\left(x_{j 2} / x_{j 1}\right) \Sigma_{T(N)}}{\Sigma_{B(N)}+\Sigma_{T(N)}}\right]-\frac{\log 2}{\pi} .
$$

Note that this expression provides the correction $\Delta \gamma$ to the growth rate. The full growth rate is given by $\gamma=\gamma_{\infty}+\Delta \gamma$ (where $\gamma_{\infty}$ is specified by eq. [9] and $\Delta \gamma$ is specified by eq. [27]). In the limit of large $N$, the ratio of the sums $\Sigma_{T(N)}$ and $\Sigma_{B(N)}$ approaches unity, almost surely, so that

$$
\frac{\Sigma_{T(N)}}{\Sigma_{B(N)}} \rightarrow 1 \quad \text { as } \quad N \rightarrow \infty .
$$

This result follows from the definition of $\Sigma_{T(N)}$ and $\Sigma_{B(N)}$ : The terms in each of these two sums is the product of ratios $x_{a} / x_{b}$, and, the terms $r_{j}$ in the first sum $\Sigma_{T(N)}$ are the inverse of those $\left(1 / r_{j}\right)$ in the second sum $\Sigma_{B(N)}$. Since the fundamental variables $x_{k}$ that make up these ratios, and the products of these ratios, are drawn from the same distribution, the above condition (33) must hold. As a consequence, the expression for the growth rate given by equation (32) approaches that of equation (27).

Corollary 2.1: Let $\sigma_{0}$ be the variance of the composite variable $\log \left(x_{j 1} / x_{j 2}\right)$ (see Theorem 2). The correction to the growth rate is positive semi-definite; specifically, $\Delta \gamma \geq 0$ and $\Delta \gamma \rightarrow 0$ in the limit $\sigma_{0} \rightarrow 0$. Further, in the limit of small variance, the growth rate approaches the asymptotic form $\Delta \gamma \rightarrow \sigma_{0}^{2} /(8 \pi)$.

Proof: In the limit of small $\sigma_{0}$, we can write $x_{j}=1+\delta_{j}$, where $\left|\delta_{j}\right| \ll 1$. In this limit, equation (27) for the growth rate becomes

$$
\Delta \gamma=\lim _{N \rightarrow \infty} \frac{1}{\pi N} \sum_{j=1}^{N} \log \left[2+\delta_{j 1}-\delta_{j 2}+\delta_{j 2}^{2}-\delta_{j 1} \delta_{j 2}+\mathcal{O}\left(\delta^{3}\right)\right]-\frac{\log 2}{\pi} .
$$

In the limit $\left|\delta_{j}\right| \ll 1$, we can expand the logarithm, and the above expression simplifies to the form

$$
\Delta \gamma=\lim _{N \rightarrow \infty} \frac{1}{2 \pi N} \sum_{j=1}^{N}\left[\delta_{j 1}-\delta_{j 2}+\delta_{j 2}^{2}-\delta_{j 1} \delta_{j 2}-\left(\delta_{j 1}-\delta_{j 2}\right)^{2} / 4+\mathcal{O}\left(\delta^{3}\right)\right] .
$$

Evaluation of the above expression shows that

$$
\Delta \gamma=\frac{1}{2 \pi}\left[\left\langle\delta_{j 2}^{2}\right\rangle-\frac{1}{4}\left\langle\left(\delta_{j 1}-\delta_{j 2}\right)^{2}\right\rangle+\mathcal{O}\left(\delta^{3}\right)\right] \rightarrow \frac{\sigma_{0}^{2}}{8 \pi} .
$$


As a result, $\Delta \gamma \geq 0$. In the limit $\sigma_{0} \rightarrow 0$, all of the $x_{j}$ approach unity and $\delta_{j} \rightarrow 0$; therefore, $\Delta \gamma \rightarrow 0$ as $\sigma_{0} \rightarrow 0$.

Although equation (27) is exact, the computation of the expectation value can be difficult in practice. As a result, it is useful to have simple constraints on the growth rate in terms of the variance of the probability distribution for the variables $x_{k}$. In particular, a simple bound can be derived:

Theorem 3: Consider the general form of Hill's equation in the unstable limit so that $h=y_{1}(\pi)=$ $\dot{y}_{2}(\pi) \gg 1$. Take the variables $r_{j}>0$. Then the growth rate is given by equation (25) and the correction term $\Delta \gamma$ obeys the constraint

$$
\Delta \gamma \leq \frac{\sigma_{0}^{2}}{4 \pi}
$$

where $\sigma_{0}^{2}$ is the variance of the distribution of the variable $\xi=\log \left(x_{j 1} / x_{j 2}\right)$, and where $x_{j}$ are independent samplings of the ratios $x_{j}=h_{j} / g_{j}$.

Proof: First we define the variable $\xi_{j}=\log r_{j}$, where $r_{j}$ is given by equation (21) above with a fixed value of $n$. In the limit of large $n$, the variable $\xi_{j}$ has zero mean and will be normally distributed. If the variables $x_{j}$ are independent, the variance of the composite variable $\xi_{j}$ will be given by

$$
\sigma_{\xi}^{2}=n \sigma_{0}^{2} .
$$

As shown below, is order to obtain $2^{N}$ terms in the sums $\Sigma_{T(N)}$ and $\Sigma_{B(N)}$, almost all of the variables $r_{j}$ will fall in the large $n$ limit; in addition, $n \rightarrow \infty$ in the limit $N \rightarrow \infty$. As a result, we can consider the large $n$ limit to be valid for purposes of evaluating the correction term $\Delta \gamma$. In practice, the variables will not be completely independent, so the actual variance will be smaller than that given by equation (38); nonetheless, this form can be used to find the desired upper limit.

Given the large $n$ limit and a log-normal distribution of $r_{j}$, the expectation values $\left\langle r_{j}\right\rangle$ and $\left\langle 1 / r_{j}\right\rangle$ are given by

$$
\left\langle r_{j}\right\rangle=\exp \left[n \sigma_{0}^{2} / 2\right]=\left\langle 1 / r_{j}\right\rangle
$$

Note that the variable $\xi_{j}$ is normally distributed, and we are taking the expectation value of $r_{j}=\exp \xi_{j}$; since the mean of the exponential is not necessarily equal to the exponential of the mean, the above expression contains the (perhaps counterintuitive) factor of 2 . As expected, larger values of $n$ allow for a wider possible distribution and result in larger expectation values. The maximum expectation values thus occur for the largest values of $n$. Since $n<N / 2$, these results, in conjunction with equation (22) imply that $\widetilde{S}$ obeys the constraint

$$
\widetilde{S}<\exp \left[N \sigma_{0}^{2} / 4\right]
$$

The constraint claimed in equation (37) then follows immediately.

Combinatorics: To complete the argument, we must show that most of the variables $r_{j}$ have a large number $n$ of factors (in the limit of large $N$ ). The number of terms in the sums $\Sigma_{T(N)}$ 
and $\Sigma_{B(N)}$ is large, namely $2^{N-1}$. Further, the ratios $r_{j}$ must contain $2 n$ different values of the variables $x_{k}$. The number $P(n \mid N)$ of different ways to choose the $2 n$ variables for $N$ cycles (and hence $N$ possible values of $x_{k}$ ) is given by the expression

$$
P(n \mid N)=\frac{N !}{(N-2 n) !(n !)^{2}} .
$$

Notice that this expression differs from the more familiar binomial coefficient because the values of $r_{j}$ depend on whether or not the $x_{k}$ factors are in the numerator or denominator of the ratio $r_{j}$. Next we note that if $n \ll N$, then the following chain of inequalities holds for large $N$ :

$$
P(n \mid N)<\frac{N^{2 n}}{(n !)^{2}} \ll 2^{N-1} .
$$

For large $N$ and $n \ll N$, the central expression increases like a power of $N$, whereas the right hand expression increases exponentially with $N$. As a result, for $n \ll N$, there are not enough different ways to choose the $x_{k}$ values to make the required number of composite ratios $r_{j}$. In order to allow for enough different $r_{j}$, the number $n$ of factors must be large (namely, large enough so that $n \ll N$ does not hold) for most of the $r_{j}$. This conclusion thus justifies our use of the large $n$ limit in the proof of Theorem 3 (where we used a log-normal form for the composite distribution to evaluate the expectation values $\left\langle r_{j}\right\rangle$ and $\left.\left\langle 1 / r_{j}\right\rangle\right)$.

Estimate: Theorem 3 provides an upper bound on the contribution of the correction term $\Delta \gamma$ to the overall growth rate. This bound depends on the value of $n$, which determines the magnitude of the expectation value $\left\langle r_{j}\right\rangle$. It is useful to have an estimate of the "typical" size of $n$. In rough terms, the value of $n$ must be large enough so that the number of possible combinations is large enough to account for the $2^{N-1}$ terms in the sums $\Sigma_{T(N)}$ and $\Sigma_{B(N)}$. For each $n$, we have $P(n \mid N)$ combinations. As a rough approximation, $n P(n \mid N)$ accounts for all of the combinations of size less than $n$. If we set $n P(n \mid N)=2^{N}$, we can solve for the ratio $n / N$ required to have enough terms, and find $n / N \approx 0.11354 \ldots \approx 1 / 9$. As a result, we expect the ratio $n / N$ to lie in the range

$$
\frac{1}{9}<\frac{n}{N}<\frac{1}{2}
$$

If we use this range of $n / N$ to evaluate the expectation value using equation (39), and estimate the growth rate, the upper end of this range provides a rigorous upper bound (Theorem 3 ). The lower end of the range only represents a rough guideline, however, since the variables are not fully independent. Nonetheless, it can be used to estimate the expectation values $\left\langle r_{j}\right\rangle$.

Notice that the upper bound is conservative. Figure 1 shows a comparison of the actual growth rate (from Theorem 2) and the bound (Theorem 3). At large variance, the actual growth rate is much less than our bound. In fact, as shown in the following section, in the limit of large variance, the growth rate $\Delta \gamma \propto \sigma_{0}$ (rather than $\left.\propto \sigma_{0}^{2}\right)$.

For this numerical experiment, we used a particular form for the $x_{k}$ variables, namely $x_{k}=$ $0.01+\left(10 a \xi_{k}\right)^{a}$, where $\xi_{k}$ is a random variable in the range $0 \leq \xi_{k} \leq 1$ and $a$ is a parameter 
that is chosen to attain varying values of $\sigma_{0}^{2}$. The exact form of the curve $\Delta \gamma\left(\sigma_{0}^{2}\right)$ depends on the distribution of the $x_{k}$. However, all of the distributions studied result in the general form shown in Figure 1, and all of the cases show the same agreement between numerical experiments and the predictions of Theorem 2 .

\subsection{Error Bounds and Estimates}

The analysis presented thus far is valid in the highly unstable limit, as defined at the beginning of this section. In other words, we have found an exact solution to the reduced problem, as encapsulated in equation (15). In this problem we are taking two limits, the long-time limit $N \rightarrow \infty$ and the "unstable" limit $h \rightarrow \infty$. In the reduced problem, as analyzed above, we take the limit $h \rightarrow \infty$ first, and then consider the long-time limit $N \rightarrow \infty$. In this subsection, we consider the accuracy of this approach by finding bounds (and estimates) for the errors in the growth rates incurred from working in the highly unstable limit. In other words, we find bounds on the difference between the results for the full problem (with large but finite $h_{k}$ ) and the reduced problem.

To assess the error budget, we write the general matrix (for the full problem) in the form

$$
\mathbf{M}=h \mathbf{B} \quad \text { where } \quad \mathbf{B} \equiv\left[\begin{array}{cc}
1 & x \phi \\
1 / x & 1
\end{array}\right] .
$$

This form is the same as the matrix of the reduced problem (in the unstable limit) except for the correction factor $\phi$ in the $(1,2)$ matrix element, where $\phi \equiv\left(1-1 / h^{2}\right)$.

Let $(\Delta \gamma)_{B}$ denote the growth rate for the matrix $\mathbf{B}$ for the full problem defined in equation (44). Similarly, let $(\Delta \gamma)_{C}$ denote the growth rate found previously for the reduced problem using the matrix $\mathbf{C}$ defined in equation (15). Through repeated matrix multiplications, the product of matrices $\mathbf{B}_{k}$ will be almost the same as for the product of matrices $\mathbf{C}_{k}$, where the difference is due to the continued accumulation of factors $\phi_{k}$. Note that the index $k$, as introduced here, denotes the cycle number, and that all of these quantities vary from cycle to cycle.

Proposition 2: The error $\varepsilon_{B C}=(\Delta \gamma)_{C}-(\Delta \gamma)_{B}$ introduced by using the reduced form of the problem (the matrices $\mathbf{C}_{k}$ ) instead of the full problem (the matrices $\mathbf{B}_{k}$ ) is bounded by

$$
0<\varepsilon_{B C}<-\frac{1}{\pi}\left\langle\log \phi_{k}\right\rangle .
$$

Proof: Since $\phi_{k}<1$, by definition, we see immediately that the growth rate for the full problem is bounded from above by that of the reduced problem, i.e.,

$$
(\Delta \gamma)_{B}<(\Delta \gamma)_{C}
$$

Next we construct a new matrix of the form

$$
\mathbf{A} \equiv \phi\left[\begin{array}{cc}
1 & x \\
1 / x & 1
\end{array}\right]=\phi \mathbf{C}
$$




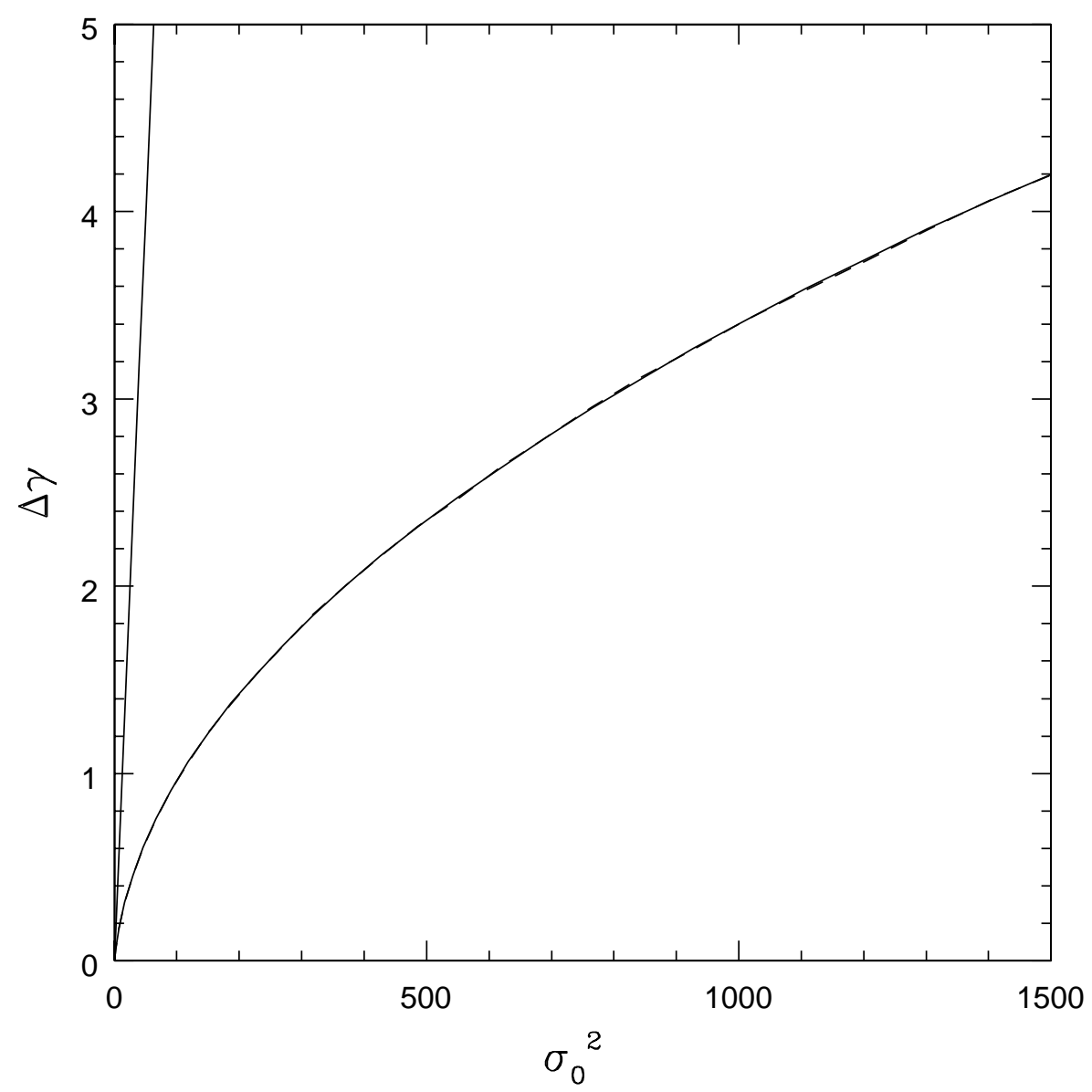

Fig. 1.- Comparison of the bound of Theorem 3 and the prediction of Theorem 2 with results from numerical experiments. All cases use matrices $\mathbf{C}_{k}$ of the form given by equation (15), where the variables $x_{k}$ are chosen according to distributions with variance $\sigma_{0}^{2}$. For each distribution, the growth rate $\Delta \gamma$ due to matrix multiplication is plotted versus the variance of the distribution of the composite variable $\xi=\log \left(x_{j} / x_{k}\right)$, where $x_{k}=y_{1 k}(\pi) / \dot{y}_{1 k}(\pi)$ and, similarly, $x_{j}=y_{1 j}(\pi) / \dot{y}_{1 j}(\pi)$. The solid curve shows the results obtained by averaging together 1000 realizations of the numerical experiments; the overlying dashed curve shows the prediction of Theorem 2 . The straight solid line shows the upper bound of Theorem 3, i.e., $\Delta \gamma \leq \sigma_{0}^{2} /(4 \pi)$. 
The products of the matrices $\mathbf{A}_{k}$ will be almost the same as those for the matrices $\mathbf{B}_{k}$, where the difference is again due to the inclusion of additional factors of $\phi_{k}$. Since the $\phi_{k}<1$, we find that the growth rate for this benchmark problem is less than (or equal to) that of the full problem, i.e., $(\Delta \gamma)_{A}<(\Delta \gamma)_{B}$. Further, the growth rate $(\Delta \gamma)_{A}$ for this new matrix can be found explicitly and is given by

$$
(\Delta \gamma)_{A}=(\Delta \gamma)_{C}+\lim _{N \rightarrow \infty} \frac{1}{\pi N} \log \left[\prod_{k=1}^{N} \phi_{k}\right]=(\Delta \gamma)_{C}+\lim _{N \rightarrow \infty} \frac{1}{\pi N} \sum_{k=1}^{N} \log \phi_{k} .
$$

Combining equations (46) and (48) shows that the growth rate for the full problem $(\Delta \gamma)_{B}$ is bounded on both sides and obeys the constraint

$$
(\Delta \gamma)_{C}+\frac{1}{\pi}\left\langle\log \phi_{k}\right\rangle<(\Delta \gamma)_{B}<(\Delta \gamma)_{C}
$$

Notice that the expectation value $\left\langle\log \phi_{k}\right\rangle<0$ since $\phi_{k}<1$. The error $\varepsilon_{B C}$ introduced by using the reduced form of the problem (the matrices $\mathbf{C}_{k}$ ) instead of the full problem (the matrices $\mathbf{B}_{k}$ ) is thus bounded by

$$
0<\varepsilon_{B C}<-\frac{1}{\pi}\left\langle\log \phi_{k}\right\rangle
$$

This bound can be made tighter by a factor of 2 . Note that the product of two matrices of the full problem has the form

$$
\mathbf{B}_{2} \mathbf{B}_{1}=\left[\begin{array}{cc}
1+\left(x_{2} / x_{1}\right) \phi_{2} & x_{1} \phi_{1}+x_{2} \phi_{2} \\
1 / x_{1}+1 / x_{2} & 1+\left(x_{1} / x_{2}\right) \phi_{1}
\end{array}\right]
$$

Thus, the product of two matrices contains only linear factors of $\phi_{k}$. As a result, we can define a new reference matrix $\widetilde{\mathbf{A}}=\phi^{1 / 2} \mathbf{C}$ that accumulates factors of $\phi_{k}$ only half as quickly as the original matrix $\mathbf{A}$ in the above argument, so that

$$
\widetilde{\mathbf{A}}_{2} \widetilde{\mathbf{A}}_{1}=\phi_{1}^{1 / 2} \phi_{2}^{1 / 2}\left[\begin{array}{cc}
1+x_{2} / x_{1} & x_{1}+x_{2} \\
1 / x_{1}+1 / x_{2} & 1+x_{1} / x_{2}
\end{array}\right]=\phi_{1}^{1 / 2} \phi_{2}^{1 / 2} \mathbf{C}_{2} \mathbf{C}_{1}
$$

The new reference matrix still grows more slowly than the matrix $\mathbf{B}$ of the full problem, but the product of $N$ such matrices accumulates only $N$ extra factors of $\phi_{k}^{1 / 2}$. Using this reference matrix in the above argument results in the tighter bound

$$
0<\varepsilon_{B C}<-\frac{1}{2 \pi}\left\langle\log \phi_{k}\right\rangle
$$

In the limit where all of the $h_{k} \gg 1, \log \phi_{k} \approx-1 / h_{k}^{2}$, and the above bound approaches the approximate form

$$
0<\varepsilon_{B C}<\frac{1}{2 \pi}\left\langle h_{k}^{-2}\right\rangle .
$$

This expression shows that the errors are well controlled. For large but finite $h_{k}$, the departure of the growth rates from those obtained in the highly unstable limit (Theorem 2) are $\mathcal{O}\left(h_{k}^{-2}\right)$. 
Given the above considerations, we can write the growth rate $(\Delta \gamma)_{B}$ for the full problem in the form

$$
(\Delta \gamma)_{B}=(\Delta \gamma)_{C}-\frac{K_{\varepsilon}}{\pi}\left\langle h_{k}^{-2}\right\rangle
$$

where $(\Delta \gamma)_{C}$ is the growth rate for the reduced problem and where $K_{\varepsilon}$ is a constant of order unity. In the limit of large $h_{k}$ (specifically, for $\log \phi_{k} \approx 1 / h_{k}^{2}$ ), the constant is bounded and lies in the range $0<K_{\varepsilon}<1 / 2$. Our numerical exploration of parameter space suggest that $K_{\varepsilon} \approx 1 / 4$ provides a good estimate for the correction term. In any case, however, the correction term depends on $h_{k}$ through the quantity $\left\langle h_{k}^{-2}\right\rangle$ and decreases with the size of this expectation value.

\subsection{Matrix Elements with Varying Signs}

We now consider the case in which the signs of the variables $r_{j}$ can be either positive or negative. Suppose that the system has equal probability of attaining positive and negative factors. In the limit $N \rightarrow \infty$, one expects the sums $\Sigma_{T(N)}, \Sigma_{B(N)} \rightarrow 0$, which would seem to imply no growth. However, two effects counteract this tendency. First, the other factor that arises in the repeated matrix multiplication diverges in the same limit, i.e.,

$$
\prod_{k}^{N}\left(2 h_{k}\right) \rightarrow \infty \quad \text { as } \quad N \rightarrow \infty .
$$

Second, the sums $\Sigma_{T(N)}$ and $\Sigma_{B(N)}$ can random walk away from zero with increasing number $N$ of cycles, where the effective step length is determined by the variance $\sigma_{0}$ defined previously. If the random walk is fast enough, the system can be unstable even without considering the diverging product of equation (56). In order to determine the stability (or instability) of the Hill's equation in this case, we must thus determine how the sums $\Sigma_{T(N)}$ and $\Sigma_{B(N)}$ behave with increasing $N$.

Theorem 4: Consider the case of Hill's equation in the unstable limit with both positive and negative signs for the matrix elements. Let positive signs occur with probability $p$ and negative signs occur with probability $1-p$. Then the general form of the growth rate is given by

$$
\Delta \gamma=\lim _{N \rightarrow \infty} \frac{1}{\pi N}\left\{\left[p^{2}+(1-p)^{2}\right] \sum_{j=1}^{N} \log \left(1+\left|\frac{x_{j 1}}{x_{j 2}}\right|\right)+2 p(1-p) \sum_{k=1}^{N} \log |1-| \frac{x_{k 1}}{x_{k 2}}||\right\}-\frac{\log 2}{\pi} .
$$

Proof: The same arguments leading to equation (32) in the proof of Theorem 2 can be used, where the signs of the ratios $x_{j 1} / x_{j 2}$ must be taken into account. If $p$ is the probability of the $x_{j}$ variables being positive, the probability of the ratio of two variables being positive will be given by $p^{2}+(1-p)^{2}$, i.e., the probability of getting either two positive signs or two negative signs. The probability of the ratio being negative is then $2 p(1-p)$. With this consideration of signs, the 
intermediate form of equation (32) is modified to take the form

$$
\begin{aligned}
\Delta \gamma+\frac{\log 2}{\pi} \approx & \frac{1}{N \pi} \sum_{j=1}^{N_{P}} \log \left[1+\frac{\left|x_{j 1} / x_{j 2}\right| \Sigma_{B(N)}+\left|x_{j 2} / x_{j 1}\right| \Sigma_{T(N)}}{\Sigma_{B(N)}+\Sigma_{T(N)}}\right] \\
& +\frac{1}{N \pi} \sum_{j=1}^{N_{Q}} \log \left[1-\frac{\left|x_{j 1} / x_{j 2}\right| \Sigma_{B(N)}+\left|x_{j 2} / x_{j 1}\right| \Sigma_{T(N)}}{\Sigma_{B(N)}+\Sigma_{T(N)}}\right],
\end{aligned}
$$

where $N_{P}$ is the number of terms where the ratios have positive signs and $N_{Q}$ is the number of terms where the ratios have negative signs. In the limit $N \rightarrow \infty$, we argue (as before) that the sums $\Sigma_{B(N)}$ and $\Sigma_{T(N)}$ approach the same value. Notice also that the two sums can be either positive or negative, but they will both have the same sign (by construction). As a result, we can divide the sums out of the expression as before. In the limit $N \rightarrow \infty$, the fraction $N_{P} / N \rightarrow p^{2}+(1-p)^{2}$ and the fraction $N_{Q} / N \rightarrow 2 p(1-p)$. After some rearrangement, we obtain the form of equation (57).

Corollary 4.1: Let $P(\xi)$ denote the probability distribution of the composite variable $\xi=x_{k} / x_{j}$, and assume that the integral $\int d \xi(d P / d \xi) \log |\xi|$ exists. Then for Hill's equation in the unstable limit, and for the case of the variables $x_{k}$ having mixed signs, in the limit of small variance the correction to the growth rate $\Delta \gamma$ approaches the following limiting form:

$$
\lim _{\sigma_{0} \rightarrow 0} \Delta \gamma=\frac{2 p(1-p)}{\pi}\left[\log \sigma_{0}+C_{0}-\log 2\right],
$$

where $C_{0}$ is a constant that depends on the probability distribution of the variables $x_{k}$.

Proof: In the limit of small $\sigma_{0}$, the variables $x_{k}$ can be written in the form $x_{k}=1+\delta_{k}$ where $\left|\delta_{k}\right| \ll 1$. To leading order, the expression of equation (57) for the growth rate becomes

$$
\Delta \gamma+\frac{\log 2}{\pi}=\lim _{N \rightarrow \infty} \frac{1}{\pi N}\left\{\left[p^{2}+(p-1)^{2}\right] \sum_{j=1}^{N} \log \left(2+\delta_{j 1}-\delta_{j 2}\right)+2 p(1-p) \sum_{k=1}^{N} \log \left|\delta_{k 1}-\delta_{k 2}\right|\right\} .
$$

In the limit of small variance $\sigma_{0} \rightarrow 0$, the variables $\delta_{k} \rightarrow 0$, and the above expression reduces to the form

$$
\Delta \gamma=\frac{2 p(1-p)}{\pi}\left[\left\langle\log \left|\delta_{k 1}-\delta_{k 2}\right|\right\rangle-\log 2\right] .
$$

We thus need to evaluate the expectation value given by

$$
\left\langle\log \left|\delta_{k}-\delta_{j}\right|\right\rangle=\int d \xi \log |\xi| \frac{d P}{d \xi}
$$

where we have defined the composite variable $\xi=\delta_{k}-\delta_{j}$. Notice that in the limit $|\delta| \ll 1$, the variance of $\xi$ is $\sigma_{0}^{2}$. Next we define a dimensionless variable $z \equiv \xi / \sigma_{0}$, so that the integral becomes

$$
I=\int d z \frac{d P}{d z} \log \left(\sigma_{0} z\right)=\log \sigma_{0} \int d z \frac{d P}{d z}+\int d z \frac{d P}{d z} \log z=\log \sigma_{0}+\int d z \frac{d P}{d z} \log z .
$$




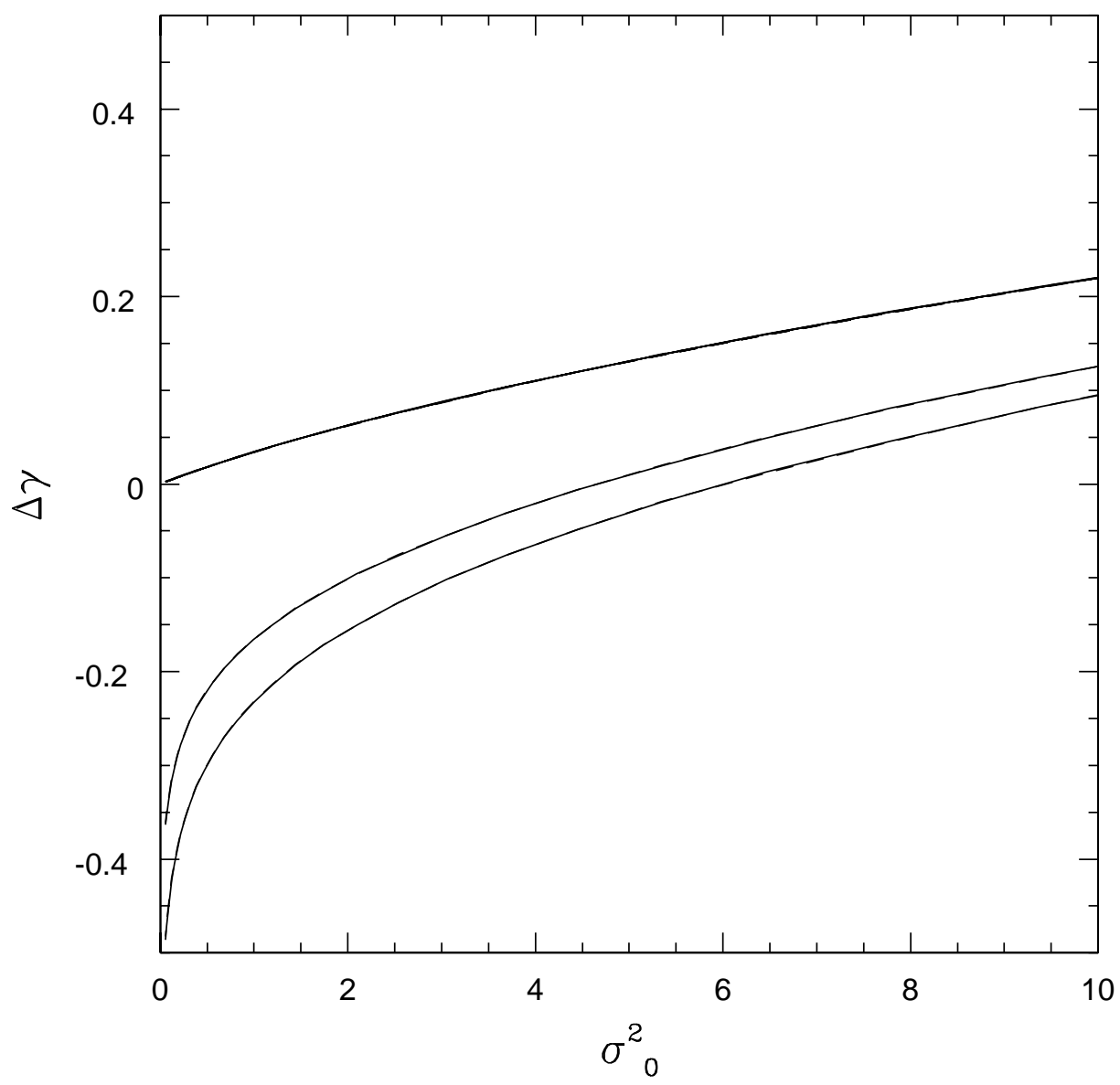

Fig. 2.- Correction $\Delta \gamma$ to the growth rate for the case in which the signs of the random variables $x_{k}$ are both positive and negative. The three curves show the results for a 50/50 distribution (bottom), 75/25 (center), and the case of all positive signs (top). For all three cases, the solid curves show the results of numerical matrix multiplication, where 1000 realizations of each product are averaged together. The overlying dashed curves, which are virtually indistinguishable, show the exact results from Theorem 4 . 


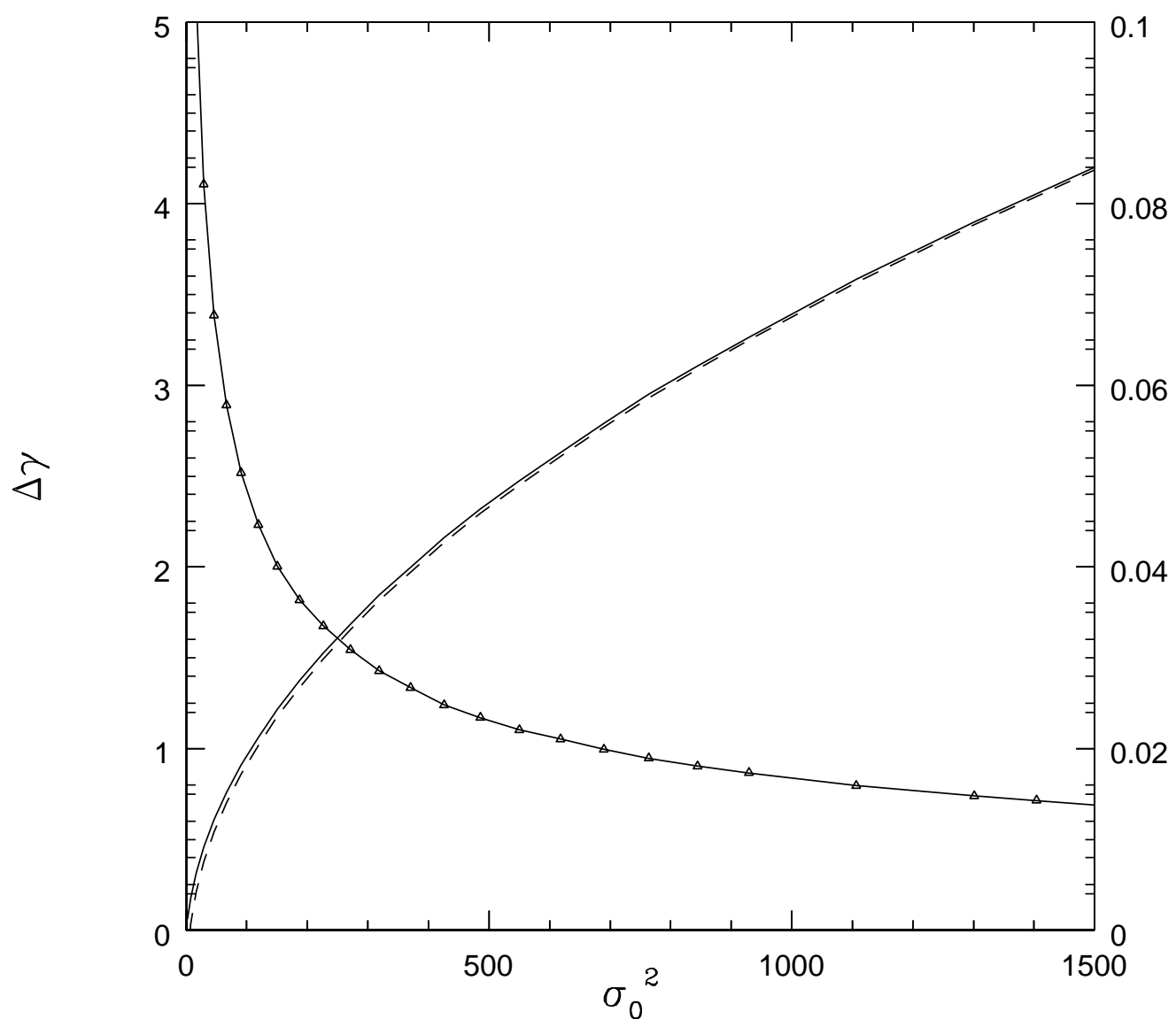

Fig. 3.- Convergence of growth rates in the limit of large variance. The increasing solid curve shows the growth rate as a function of variance for the case of all positive signs. The dashed curve shows the growth rate for the cased of mixed signs with a 50/50 sign distribution, i.e., $p=1 / 2$. The decreasing curve marked by triangles shows the difference between the two curves (where the axis on the right applies). 
As long as the differential probability distribution $d P / d z$ allows the integral in the final expression to converge, then $I=\log \sigma_{0}+C_{0}$, where $C_{0}$ is some fixed number that depends only on the shape of the probability distribution. This convergence requirement is given by the statement of the corollary, so that Corollary 4.1 holds. Notice also that in the limit of small $\sigma_{0}$, the $\log \sigma_{0}$ term dominates for any fixed $C_{0}$, so that $\Delta \gamma \sim 2 p(1-p)\left(\log \sigma_{0}\right) / \pi$.

Figure 2 shows the growth rates as a function of the variance $\sigma_{0}$ for the case of mixed signs. For the case of positive signs only, $p=1$, the correction $\Delta \gamma$ to the growth rate goes to zero as $\sigma_{0} \rightarrow 0$. For the case of mixed signs, the correction to the growth rate has the form $\Delta \gamma \propto \log \sigma_{0}$ as implied by Corollary 4.1 .

Sometimes it is useful to explicitly denote when the growth rates under consideration are the result of purely positive signs or mixed signs for the variables $x_{k}$. Here, we use the notation $\Delta \gamma_{p}$ to specify the growth rate when all the signs are positive. Similarly, $\Delta \gamma_{q}$ denotes growth rates for the case of mixed signs.

Corollary 4.2: In the limit of large variance, $\sigma_{0} \rightarrow \infty$, the growth rates for the case of positive signs only and for the case of mixed signs converge, i.e.,

$$
\lim _{\sigma_{0} \rightarrow \infty} \Delta \gamma_{q}=\Delta \gamma_{p}
$$

where $\Delta \gamma_{p}$ denotes the case of all positive signs and $\Delta \gamma_{q}$ denotes the case of mixed signs.

Proof: The difference in the growth rates for two cases is given by

$$
\Delta \gamma_{p}-\Delta \gamma_{q}=\frac{2 p(1-p)}{\pi} \lim _{N \rightarrow \infty} \frac{1}{N} \sum_{j=1}^{N}\left[\log \left(1+\left|x_{j 1} / x_{j 2}\right|\right)-\log |1-| x_{j 1} / x_{j 2}||\right]
$$

where $p$ is the probability for the sign of $x_{k}$ being positive. In the limit of large variance $\sigma_{0}^{2} \rightarrow \infty$, the ratios $\left|x_{j} / x_{k}\right|$ are almost always far from unity. Only the cases with $\left|x_{j} / x_{k}\right| \gg 1$ have a significant contribution to the sums. For those cases, however, both of the logarithms in the sums reduce to the same form, $\log \left|x_{j} / x_{k}\right|$, and hence equation (65) becomes

$$
\lim _{\sigma_{0} \rightarrow \infty} \Delta \gamma_{p}-\Delta \gamma_{q}=\frac{2 p(1-p)}{\pi}\left[\left\langle\log \left|x_{j} / x_{k}\right|\right\rangle-\left\langle\log \left|x_{j} / x_{k}\right|\right\rangle\right] \rightarrow 0
$$

As a result, equation (64) is valid.

Corollary 4.3: In the limit of large variance $\sigma_{0} \rightarrow \infty$, the growth rate $\Delta \gamma$ approaches the form given by

$$
\lim _{\sigma_{0} \rightarrow \infty} \Delta \gamma=\frac{\sigma_{0}}{\pi} C_{\infty}
$$

where $C_{\infty}$ is a constant that depends on the form of the probability distribution for the variables $x_{k}$. In general, $C_{\infty} \leq 1 / 2$.

Proof: Let the composite variable $\xi=\log \left(x_{k} / x_{j}\right)$ have a probability distribution $d P / d \xi$. Since the growth rate for the case of mixed signs converges to that for all positive signs in the limit of interest 
(from Corollary 4.2), we only need to consider the latter case (from Theorem 2). The growth rate is then given by the expectation value

$$
\Delta \gamma=\frac{1}{\pi} \int_{-\infty}^{\infty} d \xi \frac{d P}{d \xi} \log \left(1+\mathrm{e}^{\xi}\right)
$$

The integral can be separated into the domains $\xi<0$ and $\xi>0$. For the positive integral, we expand the integrand into two terms; for the negative domain, we change variables of integration so that $\xi \rightarrow-\xi$. We thus obtain the three terms

$$
\Delta \gamma=\frac{1}{\pi} \int_{0}^{\infty} d \xi \frac{d P}{d \xi} \xi+\frac{1}{\pi} \int_{0}^{\infty} d \xi \frac{d P}{d \xi} \log \left(1+\mathrm{e}^{-\xi}\right)+\frac{1}{\pi} \int_{0}^{\infty} d \xi \frac{d \widetilde{P}}{d \xi} \log \left(1+\mathrm{e}^{-\xi}\right) .
$$

In the third integral, the probability distribution $(d \widetilde{P} / d \xi)(\xi)=(d P / d \xi)(-\xi)$; the second and third terms will thus be the same since the distribution is symmetric (by construction, the composite variable $\xi$ is the difference between two variables $\log x_{k}$ drawn from the same distribution). The sum of the second two integrals is bounded from above by $\log 2$ and can be neglected in the limit of interest. In the first integral, we change variables according to $z=\xi / \sigma$, so that

$$
\Delta \gamma \rightarrow \frac{\sigma_{0}}{\pi}\langle z\rangle_{(\xi \geq 0)} \quad \text { where } \quad\langle z\rangle_{(\xi \geq 0)} \equiv \int_{0}^{\infty} d z \frac{d P}{d z} z .
$$

Since $\langle 1\rangle=1$ and $\left\langle z^{2}\right\rangle=1$, by definition, we expect the quantity $\langle z\rangle_{(\xi \geq 0)}=C_{\infty}$ to be of order unity. Further, one can show that $C_{\infty}$ as defined here is bounded from above by $1 / 2$. As a result, in this limit, we obtain a bound of the form $\pi(\Delta \gamma) \leq \sigma_{0} / 2+\log 2$. We note that the constant $C_{\infty}$ cannot be bounded from below (in the absence of further constraints placed on the probability distribution $d P / d \xi)$.

Corollary 4.4: In the limit of large variance $\sigma_{0}^{2} \gg 1$, the difference $\Delta(\Delta \gamma)$ between the growth rate for strictly positive signs and that for mixed signs takes the form

$$
\lim _{\sigma_{0} \rightarrow \infty} \Delta(\Delta \gamma)=\frac{8 p(1-p)}{\pi \sigma_{0}} C_{\Delta},
$$

where $C_{\Delta}$ is a constant that depends on the form of probability distribution, and where $p$ is the probability of positive matrix elements for the case of mixed signs.

Proof: Using the results from Theorem 2 and Theorem 4 to specify the growth rates for the cases of positive signs and mixed signs, respectively, the difference can be written in the form

$$
\Delta(\Delta \gamma)=\frac{2 p(1-p)}{\pi} \int_{-\infty}^{\infty} d \xi \frac{d P}{d \xi}\left[\log \left(1+\mathrm{e}^{\xi}\right)-\log \left|1-\mathrm{e}^{\xi}\right|\right]
$$

Next we separate the integrals into positive and negative domains and change the integration variable for the negative domain $(\xi \rightarrow-\xi)$. The integral $(I)$ then becomes

$$
I=\int_{0}^{\infty} d \xi \frac{d P}{d \xi} \log \left(\frac{1+\mathrm{e}^{-\xi}}{1-\mathrm{e}^{-\xi}}\right)+\int_{0}^{\infty} d \xi \frac{d \widetilde{P}}{d \xi}\left(\frac{1+\mathrm{e}^{-\xi}}{1-\mathrm{e}^{-\xi}}\right)
$$


where $\widetilde{P}(\xi)=P(-\xi)$. Since we are working in the large $\sigma_{0}$ limit, the variable $\xi$ will be large over most of the domain where the integrals have support, so we can expand using $\mathrm{e}^{-\xi}$ as a small parameter. In this case, the integral $I$ becomes

$$
I=2 \int_{-\infty}^{\infty} d \xi \frac{d P}{d \xi} \mathrm{e}^{-|\xi|}=2 \int_{-\infty}^{\infty} d z \frac{d P}{d z} \mathrm{e}^{-\sigma_{0}|z|},
$$

where we have made the substitution $z=\xi / \sigma$. For large $\sigma_{0}$, the decaying exponential dominates the behavior of the integrand. In the limit $\sigma \rightarrow \infty$, the exponential term decays to zero before the probability $d P / d z$ changes so that $d P / d z \rightarrow C_{\Delta}$, where $C_{\Delta}$ is a constant. The integral thus becomes $I=4 C_{\Delta} / \sigma_{0}$, and the difference between the growth rates becomes

$$
\Delta(\Delta \gamma)=\frac{8 p(1-p)}{\pi \sigma_{0}} C_{\Delta}
$$

as claimed by Corollary 4.4 .

Figure 3 illustrates the behavior implied by the last three Corollaries. In the limit of large variance, the growth rates for mixed signs and positive signs only converge (Corollary 4.2). Further, growth rates for both cases approach the form $\Delta \gamma \propto \sigma_{0}$ (as in Corollary 4.3). Finally, the difference between the growth rates for the two cases has the characteristic form $\Delta(\Delta \gamma) \propto 1 / \sigma_{0}$ (from Corollary 4.4).

Corollary 4.5: For the case of mixed signs, the crossover point between growing solutions and decaying solutions is given by the condition

$$
\left[p^{2}+(1-p)^{2}\right]\left\langle\log |1+| x_{j} / x_{k}||\right\rangle+2 p(1-p)\left\langle\log |1-| x_{j} / x_{k}||\right\rangle=\log 2 .
$$

Proof: This result follows form Theorem 4 by inspection.

Estimate for the Crossover Condition: Equation (76) is difficult to evaluate in practice. In order to obtain a rough estimate of the threshold for instability, we can consider the the $r_{j}$ to be independent variables and use elementary methods to estimate the conditions necessary for systems with mixed signs to be unstable. We first note that the sums $\Sigma_{T(N)}$ and $\Sigma_{B(N)}$ add up the composite variables $r_{j}$, which are made up of the variables $x_{j}$ (which in turn are set by the form of the original differential equation). If the signs of the variables $x_{j}$ are symmetrically distributed, then the signs of the composite variables $r_{j}$ are also symmetrically distributed. We can thus focus on the variables $r_{j}$.

Since the signs can either be positive or negative, the probability of a net excess of positive (or negative) terms is governed by the binomial distribution (which has a gaussian form in the limit of large $N$ ). The probability $P$ of having a net excess of $m$ signs is given by the distribution

$$
P(m)=\left(\pi N_{S} / 2\right)^{-1 / 2} \exp \left[-m^{2} / 2 N_{S}\right],
$$

where $N_{S}$ is the number of steps in the random walk. The sums $\Sigma_{T(N)}$ and $\Sigma_{B(N)}$ have $N_{S}=2^{N}$ steps, where $N$ is the number of cycles of the Hill's equation. 
If the net excess of signs of one type is $m$, the sums are reduced (from those obtained with purely positive variables) so that

$$
\widetilde{S}=\widetilde{S}_{0} \frac{m}{N_{S}}
$$

where $\widetilde{S}_{0}$ is the value of the composite sum obtained when the variables $x_{j}$ have only one sign.

The probability of a growing solution is given by

$$
P_{G}=\int_{m_{*}}^{\infty} P(m) d m
$$

where $m_{*}$ is the minimum number of steps needed for instability. We can write $m_{*}$ in the form

$$
m_{*}=N_{S} \mathrm{e}^{-N \pi \Delta \gamma_{0}}=\exp \left[N\left(\log (2)-\pi \Delta \gamma_{0}\right)\right],
$$

where $\Delta \gamma_{0}$ is the correction to the growth rate for the case of positive signs only.

The integral can be written in terms of the variable $\xi=m /\left(2 N_{S}\right)^{1 / 2}$ so that

$$
P_{G}=\frac{2}{\sqrt{\pi}} \int_{z_{*}}^{\infty} \mathrm{e}^{-z^{2}} d z
$$

where

$$
z_{*}=\exp \left[N\left(\frac{1}{2} \log 2-\pi \Delta \gamma_{0}\right]\right.
$$

Thus, the crossover for growth occurs under the condition

$$
\Delta \gamma_{0} \approx \log 2 /(2 \pi)
$$

Keep in mind that this result was derived under the assumption that the variables in the random walk are completely independent. We can derive the above approximate result from a simpler argument: The sums $\Sigma_{T(N)}$ and $\Sigma_{B(N)}$ random walk away from zero according to $\ell \sqrt{N_{S}}=$

$\left\langle r_{j}^{2}\right\rangle^{1 / 2} 2^{N / 2}=\exp \left[n \sigma_{0}^{2}+(N / 2) \log 2\right]$. As a result, $\widetilde{S} \approx \exp \left[n \sigma_{0}^{2}-(N / 2) \log 2\right]$ and hence $\Delta \gamma \approx$ $(n / N)\left(\sigma_{0}^{2} / \pi\right)-(\log 2) / 2 \pi$.

\subsection{Specific Results for a Normal Distribution}

In this section we consider the particular case where the composite variable $\xi=\log \left(x_{k} / x_{j}\right)$ has a normal distribution. Specifically, we let the differential probability distribution take the form

$$
\frac{d P}{d \xi}=\frac{1}{\sqrt{2 \pi} \sigma_{0}} \mathrm{e}^{-\xi^{2} / 2 \sigma_{0}^{2}}
$$

so that $\sigma_{0}^{2}$ is the variance of the distribution. In order to determine the growth rates, we must evaluate the integrals

$$
J_{ \pm}=\frac{1}{\sqrt{2 \pi} \sigma_{0}} \int_{-\infty}^{\infty} d \xi \mathrm{e}^{-\xi^{2} / 2 \sigma_{0}^{2}} \log \left|1 \pm \mathrm{e}^{\xi}\right|
$$


In the limit $\sigma_{0} \rightarrow 0$, the correction part of the growth rate $(\Delta \gamma)$ can be evaluated and has the form

$$
\lim _{\sigma_{0} \rightarrow 0} \Delta \gamma=\frac{1}{\pi}\left\{\left[p^{2}+(1-p)^{2}\right] \frac{\sigma_{0}^{2}}{8}+2 p(1-p)\left[\log \sigma_{0}-\frac{\gamma_{\mathrm{em}}}{2}\right]-3 p(1-p) \log 2\right\},
$$

where $\gamma_{\mathrm{em}}=0.577215665 \ldots$ is the Euler-Mascheroni constant. Note that for the case of positive signs only $(p=1)$, this expression reduces to the form $\Delta \gamma=\sigma_{0}^{2} /(8 \pi)$ as in Corollary 2.1. For the case of mixed signs, this expression reduces to the form $\Delta \gamma \propto \log \sigma_{0}$ from Corollary 4.1.

We can also evaluate the growth rate in the limit of large $\sigma_{0}$, and find the asymptotic form

$$
\lim _{\sigma_{0} \rightarrow \infty} \Delta \gamma=\frac{\sigma_{0}}{\sqrt{2} \pi^{3 / 2}}
$$

As a result, the constant $C_{\infty}$ from Corollary 4.3 is given by $C_{\infty}=1 / \sqrt{2 \pi}$. Note that in this limit, the growth rate is independent of the probabilities $p$ and $(1-p)$ for the variables $x_{k}$ to have positive and negative signs, consistent with Corollary 4.2. In this limit, we can also evaluate the difference between the case of positive signs and mixed signs, i.e.,

$$
\Delta \gamma_{p}-\Delta \gamma_{q}=\frac{8 p(1-p)}{\sqrt{2} \pi^{3 / 2} \sigma_{0}}
$$

Thus, the constant $C_{\Delta}$ from Corollary 4.4 is given by $C_{\Delta}=1 / \sqrt{2 \pi}$ for the case of a normal distribution. Note that although $C_{\Delta}=C_{\infty}$ for this particular example, these constants will not be the same in general.

Finally, for the case of purely positive signs, we can connect the limiting forms for small variance and large variance to construct a rough approximation for the whole range of $\sigma_{0}$, i.e.,

$$
\Delta \gamma \approx \frac{\sigma_{0}^{2} / \pi}{8+\sqrt{2 \pi} \sigma_{0}}
$$

This simple expression, which is exact in the limits $\sigma_{0} \rightarrow 0$ and $\sigma_{0} \rightarrow \infty$, has a maximum error of about $18 \%$ over the entire range of $\sigma_{0}$.

\subsection{Matrix Decomposition for Small Variance}

For completeness, and as a consistency check, we can study the growth rates by breaking the transformation matrix into separate parts. In this section we consider the case of small variance (see Appendix B for an alternate, more general, separation). In the limit of small variance, $\sigma_{0}^{2} \ll 1$, the variables $x_{k}$ only have small departures from unity and can be written in the form

$$
x_{k}=1+\delta_{k},
$$

where $\left|\delta_{k}\right| \ll 1$. The matrices of the discrete map can then be decomposed into two parts so that

$$
\mathbf{C}_{k}=\mathbf{A}_{k}+s_{k} \delta_{k} \mathbf{B}_{k}
$$


where $s_{k}= \pm 1$ is the sign of the $k$ th term, and where

$$
\mathbf{A}_{k}=\left[\begin{array}{cc}
1 & s_{k} \\
s_{k} & 1
\end{array}\right] \quad \text { and } \quad \mathbf{B}_{k}=\left[\begin{array}{cc}
0 & 1 \\
-1 & 0
\end{array}\right]
$$

The matrices $\mathbf{A}_{k}$ and $\mathbf{B}_{k}$ have simple multiplicative properties. In particular,

$$
\mathbf{A}_{j} \mathbf{A}_{k}=2 \mathbf{A}_{j} \quad \text { if } s_{j}=s_{k}, \quad \text { but } \quad \mathbf{A}_{j} \mathbf{A}_{k}=0 \quad \text { if } s_{j} \neq s_{k}
$$

and

$$
\mathbf{B}_{k}^{2}=-\mathbf{I}, \quad \mathbf{B}_{k}^{3}=-\mathbf{B}_{k}, \quad \text { and } \quad \mathbf{B}_{k}^{4}=\mathbf{I} .
$$

The product matrix $\prod \mathbf{C}_{k}$ will contain long strings of matrices $\mathbf{A}_{k}$ and $\mathbf{B}_{k}$ multiplied by each other. If any two matrices $\mathbf{A}_{k}$ have opposite signs in such a multiplication string, then the product of the two matrices will be zero and the entire string will vanish. As a result, after a large number $N$ of cycles, the only matrices that are guaranteed to survive in the product are those with only $\mathbf{B}_{k}$ factors and those with only one $\mathbf{A}_{k}$ factor. Although it is possible for strings with larger numbers of $\mathbf{A}_{k}$ to survive, it becomes increasingly unlikely (exponentially) as the number of factors increases. To a good approximation, the eigenvalue of the resulting product matrix will be given by the product

$$
\Lambda^{(N)} \approx \prod_{k=1}^{N} \delta_{k}
$$

We could correct for the possibility of longer surviving strings of $\mathbf{A}_{k}$ by multiplying by a factor of order unity; however, such a factor would have a vanishing contribution to the growth rate. The corresponding growth rate thus takes the form

$$
\Delta \gamma=\lim _{N \rightarrow \infty} \frac{2 p(1-p)}{N \pi} \sum_{k=1}^{N} \log \left|\delta_{k}\right|,
$$

where the factor $2 p(1-p)$ arises because the matrices with all positive signs lead to a zero growth rate in the limit $\sigma_{0} \rightarrow 0$, so only the fraction of the cases with mixed signs contribute. Next we note that the sum converges to an expectation value

$$
\left\langle\left|\delta_{k}\right|\right\rangle=\int d \delta \frac{d P}{d \delta} \log |\delta|
$$

Next we make the substitution $z=\delta / \sigma_{0}$ and rewrite the integral in the form

$$
\left\langle\left|\delta_{k}\right|\right\rangle=\sigma_{0} \int d z \frac{d P}{d z}+\int d z \frac{d P}{d z} \log z .
$$

In the limit of interest, $\sigma_{0} \rightarrow 0$, the first term dominates and the growth rate (to leading order) approaches the form

$$
\Delta \gamma=\frac{2 p(1-p)}{\pi} \log \sigma_{0}
$$

This form agrees with the leading order expression found earlier in Corollary 4.1 (see also Figure 2 , which shows the growth rate as a function of the variance). 


\section{HILL'S EQUATION IN THE DELTA FUNCTION LIMIT}

In many physical applications, including the astrophysical orbit problem that motivated this analysis, we can consider the forcing potential to be sufficiently sharp so that $\hat{Q}(t)$ can be considered as a Dirac delta function. For this limit, we specify the main equation considered in this section:

Definition: Hill's equation in the delta function limit is defined to have the form

$$
\frac{d^{2} y}{d t^{2}}+[\lambda+q \delta([t]-\pi / 2)] y=0
$$

where $q$ measures the strength of the forcing potential and where $\delta(t)$ is the Dirac delta function. In this form, the time variable is scaled so that the period of one cycle is $\pi$. The argument of the delta function is written in terms of $[t]$, which corresponds to the time variable mod- $\pi$, so that the forcing potential is $\pi$-periodic.

This form of Hill's equation allows for analytic solutions, as outlined below, which can be used to further elucidate the instability for random Hill's equations. In particular, in this case, we can solve for the transformation between the variables $\left(\lambda_{k}, q_{k}\right)$ that appear in Hill's equation and the derived composite variables $x_{k}$ that determine the growth rates.

\subsection{Principal Solutions}

To start the analysis, we first construct the principal solutions to equation (100) for a particular cycle with given values of forcing strength $q$ and oscillation parameter $\lambda$. The equation has two linearly independent solutions $y_{1}(t)$ and $y_{2}(t)$, which are defined through their initial conditions

$$
y_{1}(0)=1, \quad \frac{d y_{1}}{d t}(0)=0, \quad \text { and } \quad y_{2}(0)=0, \quad \frac{d y_{2}}{d t}(0)=1
$$

The first solution $y_{1}$ has the generic form

$$
y_{1}(t)=\cos \sqrt{\lambda} t \quad \text { for } \quad 0 \leq t<\pi / 2,
$$

and

$$
y_{1}(t)=A \cos \sqrt{\lambda} t+B \sin \sqrt{\lambda} t \quad \text { for } \quad \pi / 2<t \leq \pi,
$$

where $A$ and $B$ are constants that are determined by matching the solutions across the delta function at $t=\pi / 2$. We define $\theta \equiv \sqrt{\lambda} \pi / 2$ and find

$$
A=1+(q / \sqrt{\lambda}) \sin \theta \cos \theta \quad \text { and } \quad B=-(q / \sqrt{\lambda}) \cos ^{2} \theta .
$$

Similarly, the second solution $y_{2}$ has the form

$$
y_{2}(t)=\sin \sqrt{\lambda} t \quad \text { for } \quad 0<t<\pi / 2,
$$


and

$$
y_{2}(t)=C \cos \sqrt{\lambda} t+D \sin \sqrt{\lambda} t \quad \text { for } \quad \pi / 2<t \leq \pi,
$$

where

$$
C=(q / \lambda) \sin ^{2} \theta \quad \text { and } \quad D=\frac{1}{\sqrt{\lambda}}-(q / \lambda) \sin \theta \cos \theta
$$

For the case of constant parameters $(q, \lambda)$, we can find the criterion for instability and the growth rate for unstable solutions. Since the forcing potential is symmetric, $y_{1}(\pi)=d y_{2} / d t(\pi)$, from Theorem 1.1 of $[\mathrm{MW}]$. The resulting criterion for instability reduces to the form

$$
H \equiv\left|\frac{q}{2 \sqrt{\lambda}} \sin (\sqrt{\lambda} \pi)-\cos (\sqrt{\lambda} \pi)\right|>1,
$$

and the growth rate $\gamma$ is given by

$$
\gamma=\frac{1}{\pi} \log \left[H+\sqrt{H^{2}-1}\right] .
$$

In the delta function limit, the solution to Hill's equation is thus specified by two parameters: the frequency parameter $\lambda$ and the forcing strength $q$. Figure 4 shows the plane of possible parameter space for Hill's equation in this limit, with the unstable regions shaded. Note that a large fraction of the plane is unstable.

\subsection{Random Variations in the Forcing Strength}

We now generalize to the case where the forcing strength $q$ varies from cycle to cycle, but the oscillation parameter $\lambda$ is fixed. This version of the problem describes orbits in triaxial, extended mass distributions $[\mathrm{AB}]$ and is thus of interest in astrophysics. As outlined in $\S 2.2$, the solutions from cycle to cycle are connected by the transformation matrix given by equation (7). Here, the matrix elements are given by

$$
h=\cos (\sqrt{\lambda} \pi)-\frac{q}{2 \sqrt{\lambda}} \sin (\sqrt{\lambda} \pi) \quad \text { and } \quad g=-\sqrt{\lambda} \sin (\sqrt{\lambda} \pi)-q \cos ^{2}(\sqrt{\lambda} \pi / 2) .
$$

Theorem 5: Consider a random Hill's equation in the delta function limit. For the case of fixed $\lambda$, the growth rate of instability approaches the asymptotic growth rate $\gamma_{\infty}$ in the highly unstable limit $q / \sqrt{\lambda} \gg 1$, where the correction term has the following order:

$$
\gamma \rightarrow \gamma_{\infty}\left\{1+\mathcal{O}\left(\lambda / q^{2}\right)\right\}
$$

Corollary 5.1: In the delta function limit, the random Hill's equation with fixed $\lambda$ is unstable when the asymptotic growth rate $\gamma_{\infty}>0$. 


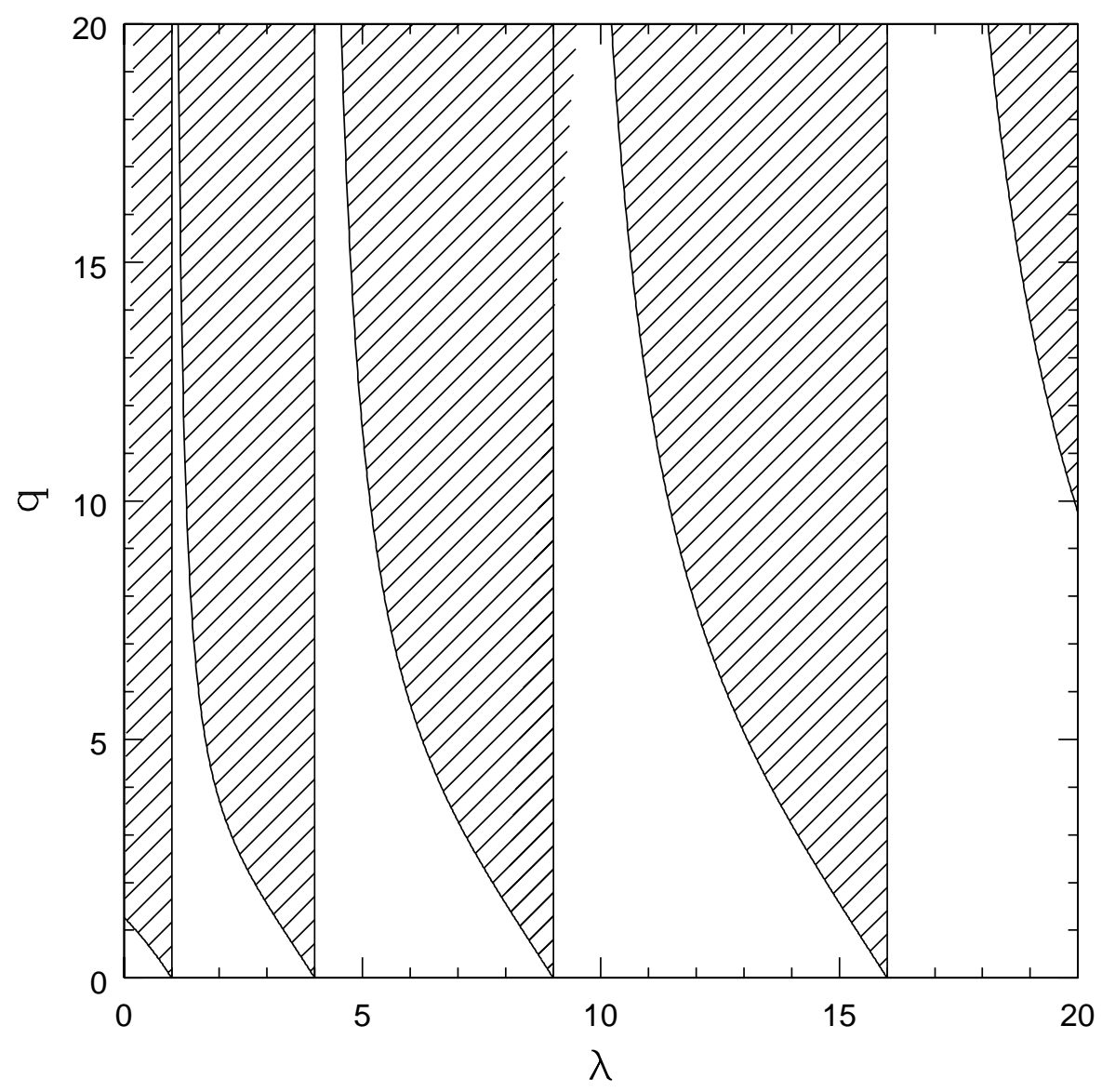

Fig. 4.- Regions of instability for Hill's equation in the delta function limit. The shaded regions show the values of $(\lambda, q)$ that correspond to exponentially growing (unstable) solutions, which represent unstable growth of the perpendicular coordinate for orbits in our triaxial potential that are initial confined to one of the principal planes. 
Remark 5.2: Note that $\gamma_{\infty}>0$ requires only that a non-vanishing fraction of the cycles are unstable.

Proof: For this version of the problem, the matrix $\mathbf{M}$ represents the transition from one cycle to the next, where the solutions are written as linear combinations of $y_{1}$ and $y_{2}$ for the given cycle. In other words, this transformation operates in the $\left(y_{1}, y_{2}\right)$ basis of solutions. However, one can also consider the purely growing and decaying solutions, which we denote here as $f_{+}$and $f_{-}$.

For a given cycle, the eigenvectors $V_{ \pm}$of the matrix $\mathbf{M}$ take the form

$$
V_{ \pm}=\left[\begin{array}{c}
1 \\
\pm g / k
\end{array}\right]
$$

where the $+(-)$ sign refers to the growing (decaying) solution. The eigenvalues have the form $\Lambda_{ \pm}$ $=h \pm k$, where $k \equiv\left(h^{2}-1\right)^{1 / 2}$. Keep in mind that $h=y_{1}(\pi)$ and $g=\dot{y}_{2}(\pi)$, and that $\Lambda_{-}=1 / \Lambda_{+}$. We can write any general solution in the form

$$
f=A V_{+}+B V_{-}
$$

where the coefficients $(A, B)$ are related to the coefficients $(\alpha, \beta)$ in the first basis through the transformation

$$
\left[\begin{array}{l}
A \\
B
\end{array}\right]=\frac{1}{2}\left[\begin{array}{cc}
1 & k / g \\
1 & -k / g
\end{array}\right]\left[\begin{array}{l}
\alpha \\
\beta
\end{array}\right]
$$

In the basis of eigenvectors, the action of the differential equation over any cycle is to amplify growing solution (eigenvector) and attenuate the decaying solution, and this action can be written as the matrix transformation

$$
\left[\begin{array}{l}
A^{\prime} \\
B^{\prime}
\end{array}\right]=\left[\begin{array}{cc}
\Lambda_{+} & 0 \\
0 & \Lambda_{-}
\end{array}\right]\left[\begin{array}{l}
A \\
B
\end{array}\right]
$$

At the end of the cycle, we can transform back to the original basis through the inverse of the transformation (114). As a result, the original matrix $\mathbf{M}$ can be decomposed into three components so that

$$
\mathbf{M}(q, \lambda)=\frac{1}{2}\left[\begin{array}{cc}
1 & 1 \\
g / k & -g / k
\end{array}\right]\left[\begin{array}{cc}
\Lambda_{+} & 0 \\
0 & \Lambda_{-}
\end{array}\right]\left[\begin{array}{cc}
1 & k / g \\
1 & -k / g
\end{array}\right]
$$

For each cycle, the values of $(q, \lambda)$ can vary. The next cycle will have a new matrix of the same general form, with the matrix elements specified by $\left(q^{\prime}, \lambda^{\prime}\right)$.

We now shift our view to the basis of eigenvectors, so that each cycle amplifies the growing solution. Between the applications of the amplification factors, the action of successive cycles "rotates" the solution according to a transition matrix of the form

$$
\mathbf{T}\left(q, \lambda ; q^{\prime}, \lambda^{\prime}\right)=\frac{1}{2}\left[\begin{array}{cc}
1 & k^{\prime} / g^{\prime} \\
1 & -k^{\prime} / g^{\prime}
\end{array}\right] \cdot\left[\begin{array}{cc}
1 & 1 \\
g / k & -g / k
\end{array}\right]=\frac{1}{2}\left[\begin{array}{cc}
1+\mathcal{R} & 1-\mathcal{R} \\
1-\mathcal{R} & 1+\mathcal{R}
\end{array}\right],
$$

where the primes denote the second cycle and where we have defined $\mathcal{R} \equiv k^{\prime} g /\left(k g^{\prime}\right)$. For the case in which successive cycles have the same values of the original parameters $(q, \lambda)$, the transition matrix $\mathbf{T}$ becomes the identity matrix (as expected). 
For simplicity, we now specialize to the case where $\lambda$ is held constant from cycle to cycle, but the forcing strength $q$ varies. We can evaluate the transition matrix for the case in which Hill's

equation lies in the delta function limit and where we also take the limit $q / \sqrt{\lambda} \gg 1$. In this regime,

$$
\mathcal{R}=1+\frac{q-q^{\prime}}{q^{\prime}} \frac{2 \sqrt{\lambda}}{q} \frac{1-2 \cos (\sqrt{\lambda} \pi)}{\sin (\sqrt{\lambda} \pi)}+\mathcal{O}\left(\frac{\lambda}{q^{2}}\right) \equiv 1+2 \delta .
$$

Note that $\mathcal{R}=1+2 \delta$ to leading order, where $\delta$ (defined through the above relation) is small compared to unity and the sign of $\delta$ can be both positive and negative. Thus, not only is the parameter $\delta$ small, but it can average to zero. Repeated iterations of the mapping lead to the $(1,1)$ matrix element growing according to the product

$$
M_{(1,1)}=\prod_{k=1}^{N}\left[\Lambda_{k}\left(1+\delta_{k}\right)\right] \approx\left[\prod_{k=1}^{N} \Lambda_{k}\right]\left[1+\sum_{k=1}^{N} \delta_{k}+\sum_{k=1}^{N} \mathcal{O}\left(\delta_{k}^{2}\right)\right]
$$

The other matrix elements are of lower order (in powers of $1 / q$ ) so that to leading order the growing eigenvalue of the product matrix is equal to the $(1,1)$ matrix element. Further, for sufficiently wellbehaved distributions of the parameter $q$, the sum of $\delta_{k}$ averages to zero as $N \rightarrow \infty$. The growth rate is thus given by

$$
\gamma=\frac{1}{\pi N} \sum_{k=1}^{N} \log \left(\Lambda_{k}\right)+\frac{1}{\pi N} \sum_{k=1}^{N} \log \left(1+\delta_{k}\right)=\gamma_{\infty}+\mathcal{O}\left(\frac{\lambda}{q^{2}}\right)
$$

The condition required for the $\delta_{k}$ to average to zero can be expressed in the form

$$
\lim _{N \rightarrow \infty} \frac{1}{N} \sum_{k=1}^{N} \frac{q^{\prime}-q}{q q^{\prime}}=\left\langle\frac{1}{q}\right\rangle-\left\langle\frac{1}{q^{\prime}}\right\rangle=0,
$$

which will hold provided that the expectation value $\langle 1 / q\rangle$ exists. This constraint is nontrivial, in that a uniform probability distribution $P(q)=$ constant that extends to $q=0$ will produce a divergent expectation value for $\langle 1 / q\rangle$. Fortunately, in the physical application that motivated this analysis, the value of $q$ is determined by the distance to the center of an orbit (appropriately weighted) so that the minimum value of $q$ corresponds to the maximum value of the distance. Since physical orbits have a maximum outer turning point (due to conservation of energy), physical orbit problems will satisfy the required constraint on the probability distribution.

\subsection{Second Matrix Decomposition}

Another way to decompose the transformation matrix is to separate it into two separate rotations, one part that is independent of the forcing strength $q$, and another that is proportional to $q$. We can thus write the matrix in the form

$$
\mathbf{M}(q, \lambda)=\mathbf{A}-\frac{q}{2 \sqrt{\lambda}} \mathbf{B} \equiv\left[\begin{array}{cc}
\cos 2 \theta & (\sin 2 \theta) / \sqrt{\lambda} \\
-\sqrt{\lambda} \sin 2 \theta & \cos 2 \theta
\end{array}\right]-\frac{q}{2 \sqrt{\lambda}}\left[\begin{array}{cc}
\sin 2 \theta & \left(2 \sin ^{2} \theta\right) / \sqrt{\lambda} \\
2 \sqrt{\lambda} \cos ^{2} \theta & \sin 2 \theta
\end{array}\right]
$$


where the second equality defines the matrices $\mathbf{A}$ and $\mathbf{B}$. With these definitions, one finds that

$$
\mathbf{A}^{N}(\theta)=\mathbf{A}(N \theta) \quad \text { and } \quad \mathbf{B}^{N}(\theta)=(2 \sin 2 \theta)^{N-1} \mathbf{B}(\theta),
$$

where we again take $\lambda$ to be constant from cycle to cycle. As a result, after $N$ cycles, the effective transformation matrix can be written in the form

$$
\mathbf{M}^{(N)}=\prod_{k=1}^{N}\left(\mathbf{A}-\frac{q_{k}}{2 \sqrt{\lambda}} \mathbf{B}\right) .
$$

In the asymptotic limit $q / \sqrt{\lambda} \rightarrow \infty$, the matrix approaches the form

$$
\mathbf{M}^{(N)}=(-1)^{N}\left[\prod_{k=1}^{N} \frac{q_{k}}{2 \sqrt{\lambda}}\right](2 \sin 2 \theta)^{N-1} \mathbf{B}(\theta) .
$$

The condition for stability takes the form $\left|\operatorname{Tr} \mathbf{M}^{(N)}\right| \geq 2$, i.e.,

$$
\left[\prod_{k=1}^{N} q_{k}\right]\left[\frac{\sin 2 \theta}{\sqrt{\lambda}}\right]^{N} \geq 1
$$

When the system is unstable, the factor on the left hand side of this equation represents the growth factor over the entire set of $N$ cycles. The growth rate $\gamma$ is thus given by

$$
\gamma=\lim _{N \rightarrow \infty} \frac{1}{\pi N} \log \left[\prod_{k=1}^{N}\left(q_{k} \frac{\sin 2 \theta}{\sqrt{\lambda}}\right)\right]=\lim _{N \rightarrow \infty} \frac{1}{\pi N} \sum_{k=1}^{N} \log \left(q_{k} \frac{\sin 2 \theta}{\sqrt{\lambda}}\right) .
$$

Since $H_{k}=q_{k}(\sin 2 \theta) / \sqrt{\lambda}$ in this asymptotic limit, the above expression for the growth rate can be rewritten in the form

$$
\gamma=\lim _{N \rightarrow \infty} \frac{1}{\pi N} \sum_{k=1}^{N} \log \left(2 H_{k}\right)=\lim _{N \rightarrow \infty} \frac{1}{N} \sum_{k=1}^{N} \gamma_{k}=\gamma_{\infty},
$$

in agreement with Theorem 5 .

\subsection{Width of Stable and Unstable Zones}

In the plane of parameters (e.g., Figure 4), the width of the stable and unstable zones can be

found for the delta function limit. In this case, the leading edge of the zone of stability is given by the condition

$$
\theta=\sqrt{\lambda} \pi=n \pi,
$$

where $n$ is an integer that can be used to label the zone in question. The beginning of the next unstable zone is given by the condition $|h|=1$. In the limit of large $q \gg 1$, the width of the stable 
regime is narrow, and the boundary will fall at $\theta=n \pi+\varphi$, where $\varphi$ is small. In particular, $\varphi$ will be smaller than $\pi / 2$, so that the angle $\theta$ will lie in either the first or third quadrant, which in turn implies that $\sin \theta$ and $\cos \theta$ have the same sign. As a result, the condition at the boundary takes the form

$$
\frac{q}{2 \sqrt{\lambda}}=\frac{1+\cos \varphi}{\sin \varphi} \approx \frac{2}{\varphi} .
$$

If we solve this expression for $\varphi$ and use the definition $\varphi=\theta-n \pi$, we can solve for the value of $\lambda$ at the boundary of the zone, i.e.,

$$
\lambda \approx \frac{n^{2}}{(1-4 / q \pi)^{2}} \approx n^{2}\left[1+\frac{8}{q \pi}+\mathcal{O}\left(q^{-2}\right)\right] .
$$

The width of the stable zone can then be expressed in the form

$$
\Delta \lambda=\frac{8 n^{2}}{\pi q}
$$

For any finite $q$, there exists a zone number $n$ such that $n^{2}>q$ and the width of the zone becomes wide. In the limit $q \rightarrow \infty$, the zones are narrow for all finite $n$.

Note that when the forcing strength $q_{k}$ varies from cycle to cycle, we can define the expectation value of the zone widths,

$$
\langle\Delta \lambda\rangle=\frac{8 n^{2}}{\pi}\left\langle\frac{1}{q_{k}}\right\rangle .
$$

This expectation value exists under the same conditions required for Theorem 5 to be valid.

\subsection{Variations in $\left(\lambda_{k}, q_{k}\right)$ and Connection to the General Case}

As outlined earlier, the growth rates $\Delta \gamma$ depend on the ratios of the principal solutions, rather than on the input parameters $\left(\lambda_{k}, q_{k}\right)$ that appear in the original differential equation (1). Since we have analytic expressions for the principal solutions in the delta function limit, we can study the relationship between the distributions of the fundamental parameters $\left(\lambda_{k}, q_{k}\right)$ and the distribution of the composite variable $\xi=\log \left(x_{k} / x_{j}\right)$ that appears in the Theorems of this paper.

As a starting point, we first consider the limiting case where $q_{k} \rightarrow \infty$ and the parameter $\lambda_{k}$ is allowed to vary. We also focus the discussion on the correction $\Delta \gamma$ to the growth rate, which depends on the ratios $x_{k}$. In this limit, using equation (110), we see the variables $x_{k}$ reduce to the simple form

$$
x_{k}=\frac{\pi}{\theta_{k}} \frac{\sin \theta_{k}}{1+\cos \theta_{k}},
$$

where $\theta_{k} \equiv \sqrt{\lambda} \pi$. In this case the distribution of $\xi=\log \left(x_{k} / x_{j}\right)$ depends only on the distribution of the angles $\theta_{k}$, which is equivalent to the distribution of $\lambda_{k}$. Since the $x_{j}$ and $x_{k}$ are drawn independently from the same distribution ( of $\theta_{k}$ ), the variance of the composite variable $\sigma_{0}^{2}=2 \sigma_{x}^{2}$, where $\sigma_{x}^{2}$ is the variance of $\log x_{k}$. 
As a benchmark case, we consider the distribution of $\theta$ to be uniformly distributed over the interval $[0,2 \pi]$. For this example,

$$
\sigma_{x}^{2}=\int_{0}^{2 \pi} \frac{d \theta}{2 \pi}\left[\log \left(\frac{\pi}{\theta} \frac{\sin \theta}{1+\cos \theta}\right)\right]^{2}-\left[\int_{0}^{2 \pi} \frac{d \theta}{2 \pi} \log \left(\frac{\pi}{\theta} \frac{\sin \theta}{1+\cos \theta}\right)\right]^{2} .
$$

Numerical evaluation indicates that $\sigma_{0} \approx 2.159$. Further, the correction to the growth rate is bounded by $\Delta \gamma \leq \sigma_{0}^{2} /(4 \pi) \approx 0.371$ and is expected to be given approximately by $\Delta \gamma \sim 0.13$. In this limit we expect the asymptotic growth rate to dominate. For example, if $q_{k} \sim 1000$, a typical value for one class of astrophysical orbits $[\mathrm{AB}]$, then $\gamma_{\infty} \approx 2$, which is an order of magnitude greater than $\Delta \gamma$. Note that in the limit of large (but finite) $q_{k}$, the corrections to equation (134) are of order $\mathcal{O}\left(1 / q_{k}\right)$, which will be small, so that the variance $\sigma_{0}^{2}$ of the composite variable $\xi$ will be nearly independent of the distribution of $q_{k}$ in this limit.

As another way to illustrate the transformation between the $\left(\lambda_{k}, q_{k}\right)$ and the matrix elements $x_{k}$, we consider the case of fixed $\lambda_{k}$ and large but finite (and varying) values of $q_{k}$. We are thus confining the parameter space in Figure 4 to a particular vertical line, which is chosen to be in an unstable band. We thus define $\theta=\sqrt{\lambda} \pi$, and the $x_{k}$ take the form

$$
x_{k}=\frac{q_{k}(\pi / \theta) \sin \theta-2 \cos \theta}{q_{k}(1+\cos \theta) / 2+(\theta / \pi) \sin \theta} .
$$

For purposes of illustration, we can make a further simplification by taking $\theta$ to have a particular value; for example, if $\theta=\pi / 2$, the $x_{k}$ are given by

$$
x_{k}=\frac{2 q_{k}}{q_{k}+1} .
$$

For this case, the relevant composite variable $\xi$ is given by

$$
\xi=\log \left[\frac{q_{k}}{q_{j}} \frac{q_{j}+1}{q_{k}+1}\right]
$$

where $q_{j}$ and $q_{k}$ are the values for two successive cycles. In the limit of large $q_{j}, q_{k} \gg 1$, the composite variable takes the approximate form $\xi \approx\left(q_{k}-q_{j}\right) /\left(q_{k} q_{j}\right)$ which illustrates the relationship between the original variables (only the $q_{k}$ in this example) and the $x_{k}$, or the composite variable $\xi$, that appear in the growth rates.

Before leaving this section, we note that the more general case of Hill's equation with a square barrier of finite width can also be solved analytically (e.g., let $\hat{Q}(t)=1 / w$ for a finite time interval of width $\Delta t=w$, with $\hat{Q}(t)=0$ otherwise). For this case, in the limit of large $q_{k}$, the solution for $h_{k}$ takes the form

$$
\left|h_{k}\right| \propto \sin \left(w q_{k}\right)^{1 / 2}\left(\frac{q_{k}}{w \lambda_{k}}\right)^{1 / 2} .
$$

In the limit of large but finite $q_{k}$ and vanishing width $w \rightarrow 0$, we recover the result from the delta function limit, i.e., the dependence on the width $w$ drops out and $\left|h_{k}\right| \propto q_{k}$. In the limit of finite $w$ 
and large $q_{k}$ [specifically, when $\left(w q_{k}\right) \ll 1$ does not hold], then $\left|h_{k}\right| \propto \sqrt{q_{k}}$. This example vindicates our expectation that large $q_{k}$ should lead to large $h_{k}$, but the dependence depends on the shape of the barrier $\hat{Q}(t)$. An interesting problem for further study is to place constraints on the behavior of the matrix elements $h_{k}$ (and $g_{k}$ ) as a function of the forcing strengths $q_{k}$ for general $\hat{Q}(t)$.

\section{DISCUSSION AND CONCLUSION}

This paper has considered Hill's equation with forcing strengths and oscillation parameters that vary from cycle to cycle. We denote such cases as random Hill's equations. Our first result is that Hill-like equations where the period is not constant, but rather varies from cycle to cycle, can be reduced to a random Hill's equation (Theorem 1). The rest of the paper thus focuses on random Hill's equations, specifically, general equations in the unstable limit (§3) and the particular cases of the delta function limit $(\S 4)$, where the solutions can be determined in terms of elementary functions.

For a general Hill's equation in the limit of a large forcing parameter, we have found general results governing instability. In all cases, the growth rates depend on the distribution of values for the elements of the transition matrix that maps the solution for one cycle onto the next. The relevant composite variable $\xi$ is determined by the principal solutions via the relation $\xi=$ $\log \left[y_{1 k}(\pi) \dot{y}_{1 j}(\pi) / \dot{y}_{1 k}(\pi) y_{1 j}(\pi)\right]$, where $k$ and $j$ denote two successive cycles; our results are then presented in terms of the variance $\sigma_{0}$ of the distribution of $\xi$. The growth rate can be separated into two parts, the asymptotic growth rate $\gamma_{\infty}$ that would result if each cycle grew at the rate appropriate for an ordinary Hill's equation, and the correction term $\Delta \gamma$ that results from matching the solutions across cycles. The asymptotic growth rate $\gamma_{\infty}$ is determined by the appropriate average of the growth rates for individual cycles (see eqs. [9] and [10]). In contrast, the correction term $\Delta \gamma$ results from a type of random walk behavior and depends on the variance of the distribution of the composite variable $\xi$ defined above.

For the case of purely positive matrix elements, the correction term $\Delta \gamma$ has a simple form (Theorem 2), and is positive semi-definite and bounded from above and below. In the limit of small variance, the correction term $\Delta \gamma \propto \sigma_{0}^{2}$, whereas in the limit of large variance, $\Delta \gamma \propto \sigma_{0}$. For all $\sigma_{0}$, the correction term to the growth rate is bounded by $\Delta \gamma \leq \sigma_{0}^{2} / 4 \pi$ (Theorem 3). A sharper bound could be obtained in the future.

For the case of matrix elements with varying signs, we have found the growth rate of instability (Theorem 4), where the results depend on the probability $p$ of the matrix elements having a positive sign. In the limit of small variance, the correction term $\Delta \gamma$ is always negative and approaches the form $\Delta \gamma \propto \log \sigma_{0}$ (unless $p=1$, where $\Delta \gamma \rightarrow 0$ in this limit). As a result, the total growth rate $\gamma=\gamma_{\infty}+\Delta \gamma$ will always be negative - and hence the system will be stable - for sufficiently small variance $\sigma_{0}$ and any admixture of mixed signs. In the opposite limit of large variance, the growth

rate for mixed signs and that for purely positive signs converge, with both cases approaching the 
form $\Delta \gamma \propto \sigma$; the difference between the growth rates for the two cases decreases as $\Delta(\Delta \gamma) \propto 1 / \sigma_{0}$.

For the delta function limit, we can find the solution explicitly for each cycle, and thus analytically define the matrix elements of the discrete map that develops the solution (eq. [7] and [110]). For the case in which only the forcing strength varies, the growth rate of the general solution approaches the asymptotic growth rate (eq. [9]), which represents the growth the solution would have if every cycle grows at the rate appropriate for a standard (non-stochastic) Hill's equation. We have calculated the widths of the stable and unstable zones for Hill's equation in the limit of delta function forcing and large growth rates, which represents a specific case of the results presented in [WK], where this specific case includes random forcing terms. Finally, we have used the analytic solutions for the delta function limit to illustrate the transformation between the original variables $\left(\lambda_{k}, q_{k}\right)$ that appear in Hill's equation and the variables $x_{k}$ that determine the growth rates $(\S 4.5)$.

Although this paper takes a step forward in our understanding of Hill's equation (in particular, generalizing it to include random forcing terms) and the multiplication of random matrices (of the particular form motivated by Hill's equation), additional work along these lines can be carried out. The analysis presented herein works primarily in the limit of large $q_{k}$, where the solutions are highly unstable, although we have bounded the errors incurred by working in this limit. Nonetheless, the case in which some cycles have stable solutions, while others have unstable solutions, should be considered in greater detail. This paper presents bounds on the correction term $\Delta \gamma$ to the growth rate, but a sharper bound could be found. In the treatment of this paper, we considered the probability distribution of the composite variable $\xi=\log \left(x_{k} / x_{j}\right)$ to be symmetric, which implies that $x_{k}$ and $x_{j}$ are independently drawn from their distribution. In future work, correlations between successive cycles can be considered and would lead to asymmetric probability distributions. Most of the results of this paper are presented in terms of the distributions of the composite variables $x_{k}$, rather than the original parameters that appear in Hill's equation; the transformation between the distributions of the $\left(\lambda_{k}, q_{k}\right)$ and the $x_{k}$ thus represents another interesting problem for future study. Another case of interest we intend to consider is the case where $\hat{Q}(t)$ takes the form a finite Fourier series. Finally, the relationship between solutions to random Hill's equations and the multiplication of random matrices should be explored in greater generality.

Random Hill's equations, and the properties of their solutions, have a wide variety of applications. The original motivation for this work was a class of orbit problems in astrophysics. In that context, many astrophysical systems - young embedded star clusters, galactic bulges, and dark matter halos - are essentially triaxial extended mass distributions. Orbits within these mass distributions are often chaotic; further, when motion is initially confined to a plane, the equation of motion for the perpendicular direction is described by a random Hill's equation. The instability explored here thus determines how quickly an orbiting body will explore the perpendicular direction. For example, this class of behavior occurs in young embedded star clusters, which begin in highly flattened configurations but quickly become rounder, in part due to the instability described here. Dark matter halos are found (numerically) to display nearly universal forms for their density distributions $[\mathrm{NF}, \mathrm{BE}]$, but an a priori explanation for this form remains lacking. Since the orbits 
of dark matter particles will be subject to the instability studied herein, random Hill's equations must play a role in the explanation. As yet another example, galactic bulges often harbor supermassive black holes at their centers; the resulting stellar orbits, including the instability considered here, play a role in feeding stars into the central black hole. Finally, we note that in addition to astrophysical applications, random Hill's equations are likely to arise in a number of other settings.

\section{Acknowledgments}

We would like to thank Charlie Doering, Gus Evrard, Divakar Vishwanath and Michael Weinstein for many useful conversations, and research students Michael Busha, Suzanne Butler, Jeff Druce, Jake Ketchum, and Eva Proszkow for performing numerical calculations that guided the initial formulation of this project. We also thank an anonymous referee for many useful comments and criticisms that improved the paper. This work was supported at the University of Michigan by the Michigan Center for Theoretical Physics; by NASA through the Spitzer Space Telescope Theoretical Research Program; and by NSF through grants CMS-0408542 and DMS-604307. Some of this work was completed at the Kavli Institute for Theoretical Physics, at U. C. Santa Barbara, and was supported in part by the National Science Foundation under Grant No. PHY05-51164.

\section{Appendix A: Astrophysical Motivation}

This Appendix outlines the original astrophysical problem that motivated this study of Hill's equation with random forcing. In the the initial setting, the goal was to understand orbits in potentials resulting from a density profile of the form

$$
\rho=\rho_{0} \frac{f(m)}{m}
$$

where $\rho_{0}$ is a density scale. This form arises in many different astrophysical contexts, including dark matter halos, galactic bulges, and young embedded star clusters. The density field is constant on ellipsoids and the variable $m$ has a triaxial form

$$
m^{2}=\frac{x^{2}}{a^{2}}+\frac{y^{2}}{b^{2}}+\frac{z^{2}}{c^{2}}
$$

where, without loss of generality, $a>b>c>0$. The radial coordinate $\xi$ is given by $\xi^{2}=x^{2}+y^{2}+z^{2}$. The function $f(m)$ is assumed to approach unity as $m \rightarrow 0$ so that the density profile approaches the form $\rho \sim 1 / m$. For this inner limit, one can find an analytic form for both the potential and the force terms $[\mathrm{AB}]$. For purposes of illustration, we write the force terms for the three spatial directions in the form

$$
\begin{gathered}
\mathcal{F}_{x}=-\frac{2 x}{F(a)} \ln \left|\frac{2 F(a) \sqrt{\Gamma}+2 \Gamma-\Lambda a^{2}}{a^{2}\left[2 F(a) \xi+\Lambda-2 a^{2} \xi^{2}\right]}\right| \\
\mathcal{F}_{y}=-\frac{2 y}{|F(b)|}\left[\sin ^{-1}\left(\frac{\Lambda-2 b^{2} \xi^{2}}{\sqrt{\Lambda^{2}-4 \xi^{2} \Gamma}}\right)-\sin ^{-1}\left(\frac{2 \Gamma / b^{2}-\Lambda}{\sqrt{\Lambda^{2}-4 \xi^{2} \Gamma}}\right)\right]
\end{gathered}
$$




$$
\mathcal{F}_{z}=-\frac{2 z}{F(c)} \ln \left|\frac{2 F(c) \sqrt{\Gamma}+2 \Gamma-\Lambda c^{2}}{c^{2}\left[2 F(c) \xi+\Lambda-2 c^{2} \xi^{2}\right]}\right| .
$$

The coefficients in the numerators are given by the following quadratic functions of the coordinates:

$$
\Lambda \equiv\left(b^{2}+c^{2}\right) x^{2}+\left(a^{2}+c^{2}\right) y^{2}+\left(a^{2}+b^{2}\right) z^{2} \quad \text { and } \quad \Gamma \equiv b^{2} c^{2} x^{2}+a^{2} c^{2} y^{2}+a^{2} b^{2} z^{2},
$$

and the remaining function $F$ is defined by

$$
F(\alpha) \equiv\left[\xi^{2} \alpha^{4}-\Lambda \alpha^{2}+\Gamma\right]^{1 / 2} .
$$

Equations (A3 - A7) define the force terms that determine the orbital motion of a test particle moving in the potential under consideration (i.e., that resulting from a triaxial density distribution of the form [A1]). The work of $[\mathrm{AB}]$ shows that when the orbit begins in any of the three principal planes, the motion is (usually) highly unstable to perturbations in the perpendicular direction. For example, for an orbit initially confined to the $x-z$ plane, the amplitude of the $y$ coordinate will (usually) grow exponentially with time. In the limit of small $y$, the equation of motion for the perpendicular coordinate simplifies to the form

$$
\frac{d^{2} y}{d t^{2}}+\omega_{y}^{2} y=0 \quad \text { where } \quad \omega_{y}^{2}=\frac{4 / b}{\sqrt{c^{2} x^{2}+a^{2} z^{2}}+b \sqrt{x^{2}+z^{2}}} .
$$

Here, the time evolution of the coordinates $(x, z)$ is determined by the orbit in the original $x-z$ plane. Since the orbital motion is nearly periodic, the $(x, z)$ dependence of $\omega_{y}^{2}$ represents a periodic forcing term. The forcing strengths, and hence the parameters $q_{k}$ appearing in Hill's equation (1), are thus determined by the inner turning points of the orbit (with appropriate weighting from the axis parameters $[a, b, c]$ ). Further, since the orbit in the initial plane often exhibits chaotic behavior, the distance of closest approach of the orbit, and hence the strength $q_{k}$ of the forcing, varies from cycle to cycle. The orbit also has outer turning points, which provide a minimum value of $\omega_{y}^{2}$, which defines the unforced oscillation frequency $\lambda_{k}$ appearing in Hill's equation (1). As a result, the equation of motion (A8) for the perpendicular coordinate has the form of Hill's equation, where the period, the forcing strength, and the oscillation frequency generally vary from cycle to cycle.

\section{Appendix B: Growth Rate for an Ancillary Matrix}

In this Appendix, we separate the transformation matrix for the general case (not in the limit of small variance) and find the growth rate for one of the matrices. We include this result because examples where one can explicitly find the growth rates (Lyapunov exponents) for random matrices are rare. Specifically, the transition matrix can be written in the form given by equation (91), where the second term in the sum has the form

$$
s_{k}\left(x_{k}-1\right) \mathbf{B}_{k} \quad \text { where } \quad \mathbf{B}_{k}=\left[\begin{array}{cc}
0 & 1 \\
-1 / x_{k} & 0
\end{array}\right] .
$$


Note that any pair of matrices $\mathbf{A}_{k}$ with opposite signs will vanish, and so will all subsequent products.

The products of the second term (the matrices $\mathbf{B}_{k}$ along with the leading factor) have a well-defined growth rate:

Proposition 3: The growth rate of matrix multiplication for the matrix $\mathbf{M}_{k}=\left(x_{k}-1\right) \mathbf{B}_{k}$ is given by

$$
\gamma_{B}=\lim _{N \rightarrow \infty} \frac{1}{2 \pi N}\left\{\sum_{k=1}^{N} \log \left|x_{k}-1\right|+\sum_{j=1}^{N} \log \left|1 / x_{j}-1\right|\right\}
$$

Proof: The products of the matrices $\mathbf{B}_{k}$ follow cycles as shown by the first three nontrivial cases:

$$
\mathbf{B}_{2} \mathbf{B}_{1}=\left[\begin{array}{cc}
-1 / x_{1} & 0 \\
0 & -1 / x_{2}
\end{array}\right], \quad \mathbf{B}_{3} \mathbf{B}_{2} \mathbf{B}_{1}=\left[\begin{array}{cc}
0 & -1 / x_{2} \\
1 /\left(x_{1} x_{3}\right) & 0
\end{array}\right]
$$

and

$$
\mathbf{B}_{4} \mathbf{B}_{3} \mathbf{B}_{2} \mathbf{B}_{1}=\left[\begin{array}{cc}
1 /\left(x_{1} x_{3}\right) & 0 \\
0 & 1 /\left(x_{2} x_{4}\right)
\end{array}\right] .
$$

Thus, the even products of the matrices are diagonal matrices, whereas the odd products produce matrices with only off-diagonal elements. As a result, the product matrix will approach the form

$$
\mathbf{M}^{(N)} \sim\left(\prod_{k=1}^{N}\left(x_{k}-1\right)\right)\left[\begin{array}{cc}
P_{\text {odd }} & 0 \\
0 & P_{\text {even }}
\end{array}\right] \quad \text { or } \quad \mathbf{M}^{(N)} \sim\left(\prod_{k=1}^{N}\left(x_{k}-1\right)\right)\left[\begin{array}{cc}
0 & -P_{\text {even }} \\
P_{\text {odd }} & 0
\end{array}\right],
$$

where we have defined

$$
P_{\text {odd }} \equiv \prod_{k=1, \text { odd }}^{N} \frac{1}{x_{k}} \quad \text { and } \quad P_{\text {even }} \equiv \prod_{k=2, \text { even }}^{N} \frac{1}{x_{k}}
$$

For $N$ even (odd), the eigenvalues are $\Lambda=P_{\text {even }}, P_{\text {odd }}\left(\Lambda= \pm i \sqrt{P_{\text {even }} P_{\text {odd }}}\right)$. Since $\left|P_{\text {even }}\right|=\left|P_{\text {odd }}\right|$ in the limit $N \rightarrow \infty$, the eigenvalues (and hence the growth rates) have the same magnitudes in either case. To compute the growth rate $\gamma_{B}$, we need to account for the fact that only half of the factors (either the even or odd terms) appear in the products $P_{\text {odd }}$ and $P_{\text {even }}$. After some rearrangement, we obtain equation (B2).

\section{REFERENCES}

[AS] Abramowitz, M., \& Stegun, I. A. 1970, Handbook of Mathematical Functions (New York: Dover)

[AB] Adams, F. C., Bloch, A. M., Butler, S. C., Druce, J. M., \& Ketchum, J. A. 2007, Orbits and Instabilities in a Triaxial Cusp Potential, Astrophys. J., in press 
[BD] Baik, J, Deift, P. \& Strahov, E. 2003, Products and ratios of polynomials of random Hermitian matrices, J. Math. Phys. 44, 3657-3670

[BE] Busha, M. T., Evrard, A. E., Adams, F. C., \& Wechsler, R. H. 2005, The ultimate halo mass in a $\Lambda$ CDM universe, Monthly Notices R. Astron. Soc., 363, L11

[BT] Binney, J., \& Tremaine, S. 1987, Galactic Dynamics (Princeton: Princeton Univ. Press)

[BL] Bougerol, P., \& Lacroix, J. 1985, Products of Random Matrices with Applications to Schrödinger Operators (Boston: Birkhäuser)

[DE] Deift, P. 1999, Orthogonal Polynomials and Random Matrices, A Riemann- Hilbert Approach (CIMS Lecture Notes, NYU)

[FU] Furstenberg, H. 1963 Non-commuting random products, Trans. Amer. Math. Soc. 108, 377-428

[FK] Furstenberg, H., \& Kesten, H. 1960, Products of random matrices, Ann. Math. Stat., 31, 457

[HI] Hill, G. W. 1886, On the part of the motion of the lunar perigee which is a function of the mean motions of the Sun and Moon, Acta. Math., 8, 1

[LR] Lima, R., \& Rahibe, M. 1994, Exact Lyapunov exponent for infinite products of random matrices, J. Phys. A: Math. Gen., 27, 3427

[MW] Magnus, W., \& Winkler, S. 1966, Hill's Equation (New York: Wiley)

[ME] Mehta, M. 1991, Random Matrices, 2nd Edition (Academic Press, Boston)

[NF] Navarro, J. F., Frenck, C. S., \& White, S.D.M. 1997, A universal density profile from hierarchical clustering, Astrophys. J., 490, 493

[VI] Viswanath, D. 2000 Random Fibonacci sequences and the number 1.13198824...Mathematics of Computation 69 1131-1155

[WK] Weinstein, M. I., \& Keller, J. B. 1987, Asymptotic behavior of stability regions for Hill's equation, SIAM J. Ap. Math., 47, 941 